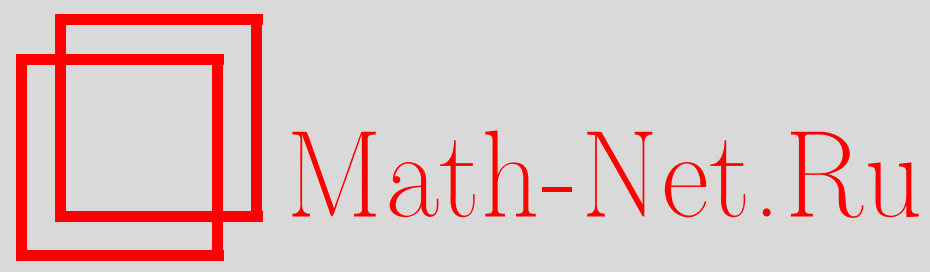

А. М. Чеботарев, С. Ю. Шустиков, Достаточные условия консервативности минимальной квантовой динамической полугруппы, Матем. заметки, 2002, том 71, выпуск 5, 761781

DOI: https://doi.org/10.4213/mzm384

Использование Общероссийского математического портала Math-Net.Ru подразумевает, что вы прочитали и согласны с пользовательским соглашением http://www.mathnet.ru/rus/agreement

Параметры загрузки:

IP : 54.209 .52 .79

26 апреля 2023 г., 09:57:38

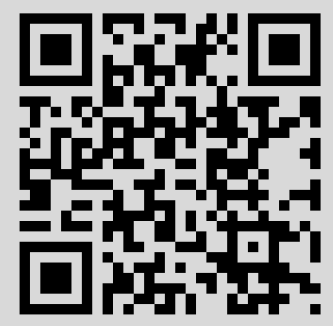




\title{
ДОСТАТОЧНЫЕ УСЛОВИЯ КОНСЕРВАТИВНОСТИ МИНИМАЛЬНОЙ КВАНТОВОЙ ДИНАМИЧЕСКОЙ ПОЛУГРУППЫ
}

\author{
А. М. Чеботарев, С. Ю. Шустиков
}

\begin{abstract}
Получены достаточные условия консервативности минимальной квантовой динамической полугруппы (КДП) для класса задач квантовой оптики с гамильтонианами, являющимися самосопряженными полиномами конечного порядка по операторам рождения и уничтожения. Порядок гамильтониана может быть больше, чем порядок вполне положительной части генератора КДП. Свойство консервативности (или унитальности) минимальной КДП обеспечивает единственность решения соответствующего уравнения марковской эволюции, т.е. в унитальном случае формальный генератор единственным образом определяет КДП, при этом КДП сохраняет единичную наблюдаемую в гейзенберговском представлении или след начального состояния в шрёдингеровском представлении. Аналогами условия консервативности для классических уравнений марковской эволюции (уравнение теплопроводности и уравнение Колмогорова-Феллера) являются условия отсутствия взрыва или ухода траекторий на бесконечность.

Библиографория: 24 названия.
\end{abstract}

Введение. Пусть $H$ - сепарабельное гильбертово пространство с полуторалинейным скалярным произведением $(\cdot, \cdot)$ и $\mathscr{B}(\mathscr{H})$ - алгебра фон Неймана, состоящая из всех ограниченных операторов, действуюших в $\mathscr{H}$. Формальньй генератор $\mathscr{L}(\cdot)$,

$$
\mathscr{L}(B)=\Phi(B)-\Phi(I) \circ B+i[H, B], \quad \Phi(B)=\sum_{k} \Phi_{k}^{*} B \Phi_{k}, \quad A \circ B=\frac{1}{2}(A B+B A),
$$

квантовой динамической полугрупшы $P_{t}(B)=e^{t \mathscr{L}}(B)$ на алгебре $\mathscr{B}(\mathscr{H})$ называется регулярны.м, если он однозначно определяет полугрупу $P_{t}(\cdot)$. Предполагается, что коэффициентами $\mathscr{L}(\cdot)$ являются плотно определенный симметричньй оператор $H$, действующий в $\mathscr{H}$ (гамильтониан), и нормальное вполне положительное отображение $\Phi(\cdot)$ (диссипативная часть), действующее в $\mathscr{B}(\mathscr{H})$. Математические трудности теории уравнений с неограниченными генераторами связаны, например, с тем, что операторы

Работа выполнена при поддержке фонда INTAS, грант № 99-0545. 
$\Phi(B) \leqslant\|B\| \Phi(I)$ могут оказаться незамыкаемьпи в $\mathscr{H}$ даже в том случае, если оператор $B$ ограничен, а $\Phi(I)$ самосопряжен ${ }^{1}$.

Структура генератора $\mathscr{L}(\cdot)$ сходна со структурой классического уравнения Колмогорова-Феллера, и аналогично классическому случаю квантовое уравнение марковской эволюции

$$
\frac{d}{d t} P_{t}(B)=\mathscr{L}\left(P_{t}(B)\right), \quad P_{0}(B)=B,
$$

при определенных условиях имеет минимальное решение, называемое минимальной квантовой динамической полугруппой. Кроме того, так же, как и в классическом случае (см. [2]-[4]), если минимальная КДП консервативна или, что то же самое, унитальн $a^{2}$, она является единственным решением соответствующего уравнения марковской эволюции; это обеспечивает регулярность формального генератора $\mathscr{L}(\cdot)$.

Резольвентные критерии регулярности генераторов КДП [2]-[7] эффективны для уравнений, у которых гамильтонова составляющая генератора подчинена его вполне положительной части. Это условие не выполняется в некоторых задачах квантовой оптики. Другим недостатком резольвентных методов является невозможность их применения к уравнениям с генераторами, зависящими от времени. В физических задачах такая зависимость возникает при использовании представления взаимодействия. В настоящей статье предлагается способ устранения указанных недостатков. Важную роль для нас сыграли работы [4]-[7], в которых уравнение для КДП рассматривается в интегральной форме, позволяющей исключить из рассмотрения операции с незамыкаемыми квадратичными формами. В этих работах, в частности, получены достаточные условия, при которых существует алгебра состояний $\mathscr{T}\left(\mathscr{H}_{\Lambda}\right)$, на которой квадратичная форма $\Phi_{*}(B)[\cdot]$ ограничена для любого $B \in \mathscr{B}(\mathscr{H})$ (и, следовательно, замыкаема), инвариантная относительно оператора эволюции $T_{t}(\cdot)$.

Основная идея настоящей статьи состоит в подходящем выборе оператора $\Lambda \geqslant \Phi(I)$

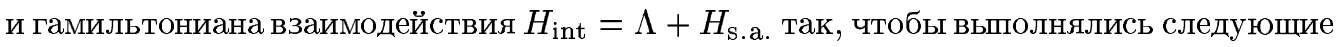
условия:

$$
\begin{array}{cc}
H=H_{\text {s.a. }}+H_{s}, \quad-H_{\text {s.a. }} \leqslant \mu H_{\text {int }}^{\varepsilon}, \quad \delta \Lambda^{2} \leqslant H_{\text {int }}^{2} \leqslant \nu \Lambda^{2}, \\
\mathscr{L}_{0}(\Lambda) \leqslant c_{0} \Lambda, \quad \Phi\left(H_{\text {s.a. }}\right) \leqslant c_{1} \Lambda, \quad \Phi\left(H_{\text {int }}^{\varepsilon}\right) \leqslant c_{2} \Lambda
\end{array}
$$

для некоторых положительных $\delta, c, \mu$ и $\nu$ и показателя степени $\varepsilon \in(0,1)$, где $\mathscr{L}_{0}(\cdot)=$ $\mathscr{L}(\cdot)-i\left[H_{\mathrm{int}}, \cdot\right]$, т.е. $\mathscr{L}_{0}(X)=\Phi(X)-G_{0}^{*} X-X G_{0}, G_{0}=\frac{1}{2} \Phi(I)+i\left(H_{s}-\Lambda\right), H_{s}-$ симметричный оператор, а $H_{\text {s.a. }}-$ самосопряженньй, $H_{\text {int }}=H_{\text {s.a. }}+\Lambda$-положительньй

\footnotetext{
${ }^{1}$ Пусть $\mathscr{H}=\ell_{2}, \Phi(B)=a B a^{\dagger}$, где $a$ и $a^{\dagger}-$ операторы уничтожения и рождения, действуюшие в $\ell_{2}$, а $B$ - ограниченный оператор в $\ell_{2}$. Если $\pi$ - проектор на вектор $\psi=\left\{1,2^{-1 / 2-\varepsilon}, 3^{-1 / 2-\varepsilon}\right.$, $\ldots\} \in \ell_{2}$, то оператор $\Phi(\pi)$ и соответствующая ему квадратичная форма $\Phi_{*}(\pi)[h]=\left|\left(\psi, a^{\dagger} h\right)_{\ell_{2}}\right|^{2}$ незамыкаемы в $\ell_{2}$ [1]. Однако в гильбертовом пространстве со скалярным произведением $\left(\Lambda^{1 / 2} g\right.$, $\left.\Lambda^{1 / 2} h\right)_{\ell_{2}}$ квадратичные формы $\Phi_{*}(B)[\cdot]$ ограничены и замыкаемы, если $\Lambda \geqslant \Phi(I)+I$. Нижний индекс * обозначает квадратичную форму.

${ }^{2}$ КДП в гейзенберговском представлении $P_{t}(\cdot)$ и КДП в шрёдингеровском представлении $T_{t}(\cdot)$ двойственны относительно скалярного произведения $\left\langle T_{t}(\rho), B\right\rangle \stackrel{\text { def }}{=} \operatorname{Tr}\left\{T_{t}(\rho) B\right\}=\operatorname{Tr}\left\{\rho P_{t}(B)\right\}=$ $\left\langle\rho, P_{t}(B)\right\rangle$, где $\rho$ - элемент алгебры $\mathscr{T}(\mathscr{H})$ операторов с конечным следом. Очевидно, что если $P_{t}(I)=I$, то $\operatorname{Tr} T_{t}(\rho)=\operatorname{Tr} \rho$. Унитальная КДП сохраняет след начального состояния в шрёдингеровском представлении и единичную наблюдаемую в гейзенберговском представлении.
} 
и самосопряженный, $H_{0}=H_{s}-\Lambda$ - симметричньй оператор. Предполагается, что $\operatorname{dom} \Lambda$ является существенной областью определения для оператора $H_{\text {s.a. }}$ и $H_{\text {int }}$.

В статье доказано, что в этом случае существуют минимальные двойственные КДП $P_{t}(\cdot)$ и $T_{t}(\cdot)$ и алгебра состояний $\mathscr{T}\left(\mathscr{H}_{\Lambda}\right)$, порождаемая гильбертовьм пространством $\mathscr{H}_{\Lambda}=\Lambda^{-1 / 2} \mathscr{H}$, инвариантная относительно $T_{t}(\cdot)$. Унитальность КДП $P_{t}(\cdot)=e^{t \mathscr{L}}(\cdot)$ следует из унитальности унитарно эквивалентного коцикла $P_{t, s}(\cdot): P_{t, s}(B)=$ $U_{t}^{*} P_{t-s}\left(U_{s} B U_{s}^{*}\right) U_{t}, U_{t}=e^{i t H_{\mathrm{int}}}$, с генератором $\mathscr{L}_{t}(B)=U_{t}^{*} \mathscr{L}_{0}\left(U_{t} B U_{t}^{*}\right) U_{t}$, так как $P_{t, s}(I)=I$ тогда и только тогда, когда $P_{t}(I)=I$. В статье доказано, что коцикл $P_{t, s}(\cdot)$ унитален, если можно подобрать пару $\left(\Lambda, H_{\mathrm{int}}\right)$ так, чтобы условия $(0.1),(0.2)$ были выполнены.

Статья имеет следующую структуру. В п. 1 рассматривается конструкция минимального решения уравнения с переменными операторными коэффициентами и доказываются достаточные условия его регулярности. В п. 2 доказьвается, что при условиях $(0.1),(0.2)$ алгебра $\mathscr{T}\left(\mathscr{H}_{\Lambda}\right)$ инвариантна относительно $T_{t}(\cdot)$ и полугруппа $P_{t}(\cdot)$ унитальна. В п. 3 описываются генераторы, используемые в квантовой оптике. Во многих случаях $H_{s}$ и $\Phi(\cdot)$ имеют второй порядок по операторам рождения и уничтожения, поэтому нетрудно удовлетворить всем условиям (0.1), (0.2). Открытой проблемой, однако, остается доказательство самосопряженности главной части оператора $H$, которая, как правило, имеет более высокий порядок, чем вполне положительная часть генератора, и не ограничена снизу. В п. 4 рассматриваются примеры уравнений квантовой оптики, большинство из которых регулярны. В п. 5 обосновывается достаточное условие нарушения регулярности.

1. Условия, достаточные для консервативности. Рассмотрим уравнение марковской эволюции

$$
\frac{\partial}{\partial t} P_{t, \tau}(B)=\mathscr{L}_{t}\left(P_{t, \tau}(B)\right),\left.\quad P_{t, \tau}(B)\right|_{\tau=t}=B
$$

с генератором $\mathscr{L}_{t}(\cdot)$, зависящим от времени,

$$
\begin{gathered}
\mathscr{L}_{t}(B)=\Phi_{t}(B)-G_{t}^{*} X-X G_{t}, \quad G_{t}=\frac{1}{2} \Phi_{t}(I)+i H_{t}, \quad \Phi_{t}(B)=\sum_{k} \Phi_{k, t}^{*} B \Phi_{k, t}, \\
\Phi_{t}(I)=\sum_{k} \Phi_{k, t}^{*} \Phi_{k, t} .
\end{gathered}
$$

Операторы $\Phi_{k, t}$ предполагаются замкнутыми; при этом $\Phi_{k, t}^{* *}=\Phi_{k, t}$. Свойство коцикличности семейства $P_{t, \tau}(\cdot)$ состоит в том, что $P_{t, \tau}\left(P_{\tau, s}(B)\right)=P_{t, s}(B)$.

В дальнейшем предполагается, что существует ограниченно обратимьй самосопряженньй оператор $\Lambda \geqslant \Phi_{t}(I) \geqslant 0$ такой, что для каждого $t \in \mathbb{R}_{+}$вполне положительное отображение $A_{t}(\cdot)=\Lambda^{-1 / 2} \Phi_{t}(\cdot) \Lambda^{-1 / 2}$ является ограниченным и нормальным [8]. Ограниченность $A_{t}(\cdot)$ следует из неравенства $\Lambda \geqslant \Phi_{t}(I)$, и в дальнейшем нам нужно предположить, что оно нормально ${ }^{3}$. Кроме того, мы предполагаем, что существует

\footnotetext{
${ }^{3}$ Например, из теоремы Крауза [9] следует, что любое нормальное ограниченное вполне положительное отображение $A_{t}(X)$ на $\mathscr{B}(\mathscr{H})$ в сепарабельным гильбертовом пространстве $\mathscr{H}$ может быть представлено как сумма $A_{t}(X)=\sum_{k} A_{k, t}^{*} X A_{k, t}$. Отсюда следует представление неограни-
} 
сильно непрерывное семейство сжимающих операторов $W_{t, s} \in \mathscr{B}(\mathscr{H})(t \geqslant s)$ с генератором $-G_{t}$ такое, что

$$
\frac{\partial}{\partial t} W_{t, s}=-W_{t, s} G_{t}, \quad \frac{\partial}{\partial s} W_{t, s}=G_{s} W_{t, s} .
$$

Семейство $W_{t, \tau}$ обладает свойством левого мультипликативного коцикла $W_{t, \tau} W_{\tau, s}=$ $W_{t, s}$, а сопряженное семейство $W_{t, \tau}^{*}$ является правы.м коциклом. В дальнейшем основ-

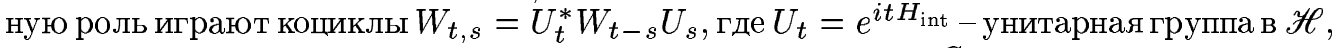
порождаемая самосопряженным оператором $H_{\mathrm{int}}$, a $W_{t}=e^{-G_{0} t}-$ сильно непрерывная сжимающая полугруппа, порождаемая аккреативным оператором $-G_{0}$; в этом случае $G_{t}=U_{t}^{*} G_{0} U_{t}$.

Будем считать, что операторы $\left\{G_{t}\right\}_{t \in \mathbb{R}_{+}}$имеют общую плотную существенную область определения $\mathscr{D}=\operatorname{dom} G_{t}^{N}$ такую, что при некотором $N \geqslant 1$ и любых $t>0$ выполнены

(А) предполохсения об областях определения: $\mathscr{D} \subseteq \operatorname{dom} \Lambda^{1 / 2} \subseteq \operatorname{dom} \Phi_{*, t}(I)[\cdot]$ и

$2 \operatorname{Re}\left(\psi, G_{t} \psi\right)=\left(\psi,\left(G_{t}^{*}+G_{t}\right) \psi\right)=\sum_{k}\left\|\Phi_{k, t} \psi\right\|^{2} \stackrel{\text { def }}{=} \Phi_{*, t}(I)[\psi] \quad \forall \psi \in \mathscr{D}$,

т.е. $W_{t, s}: \mathscr{D} \rightarrow \mathscr{D}$ и операторы $H_{t}$ симметричны на $\mathscr{D}$, где $\Phi_{*, t}(B)[\cdot]-$ положительная квадратичная форма на $\operatorname{dom} \Lambda^{1 / 2}$, сопоставляемая положительному оператору $\Phi_{t}(B)$,

$$
\Phi_{*, t}(B)[\psi] \stackrel{\text { def }}{=} \sum_{k}\left(\Phi_{k, t} \psi, B \Phi_{k, t} \psi\right) \leqslant\|B\| \cdot \sum_{k}\left\|\Phi_{k, t} \psi\right\|^{2}=\|B\| \cdot\left\|\Phi_{t}(I)^{1 / 2} \psi\right\|^{2} ;
$$

(В) условия непрерывности: семейство операторов $W_{t, s}$ ограничено и сильно непрерывно по $s$ и $t$ в гильбертовом пространстве $\mathscr{H}_{1}$ со скалярным произведением $(h, g)_{1}=\left(\Lambda^{1 / 2} h, \Lambda^{1 / 2} g\right)$, а семейство $A_{t}(\cdot)=\Lambda^{-1 / 2} \Phi_{t}(\cdot) \Lambda^{-1 / 2}$ состоит из вполне положительных ограниченных нормальных отображений таких, что функция

$$
f_{t}=\sum_{k}\left\|\Phi_{k, t} \Lambda^{-1 / 2} \psi\right\|^{2}=A_{t}(I)[\psi]
$$

непрерывна по переменной $t$ для любого $\psi \in \mathscr{H}$.

Пусть $W_{t, \tau}^{*} B W_{t, \tau} \stackrel{\text { def }}{=} V_{t, \tau}(B) \leqslant\|B\| \cdot V_{t, \tau}(I)$. Поскольку операторы $W_{t, s}$ сжимающие, то очевидно, что $0 \leqslant V_{t, \tau}(I) \leqslant I$, и из тождества

$$
\begin{aligned}
I-V_{t, \tau}(I) & =\int_{\tau}^{t} d s \frac{\partial}{\partial s} V_{t, s}(I)=\int_{\tau}^{t} d s W_{t, s}^{*}\left(G_{s}^{*}+G_{s}\right) W_{t, s} \\
& =\int_{\tau}^{t} d s W_{t, s}^{*} \Phi_{s}(I) W_{t, s}=\int_{\tau}^{t} d s V_{t, s} \Phi_{s}(I),
\end{aligned}
$$

ченного вполне положительного нормального отображения $\Phi_{t}(\cdot)$ [1]:

$$
\Phi_{t}(X)=\sum_{k} \Phi_{k, t}^{*} X \Phi_{k, t}, \quad \Phi_{k, t}=A_{k, t} \Lambda^{1 / 2}, \quad A_{t}(I)=\sum_{k} A_{k, t}^{*} A_{k, t} \in \mathscr{B}(\mathscr{H}) .
$$


справедливого в силу симметричности $H$ на плотном множестве $\mathscr{D}$, следует оценка

$$
0 \leqslant \int_{\tau}^{t} d s V_{t, s} \Phi_{s}(I) \leqslant I
$$

Если выполнены предположения $(\mathbf{A})$ и $(\mathbf{B})$, то для любого ограниченного сильно непрерьвного семейства операторов $X_{s}$ семейство квадратичных форм

$$
\Phi_{*, s}\left(X_{s}\right)\left[W_{t, s} \psi\right]=A_{*, s}\left(X_{s}\right)\left[\psi_{t, s}\right], \quad \psi_{t, s}=\Lambda^{1 / 2} W_{t, s} \Lambda^{-1 / 2} \psi \in \mathscr{H} \quad \forall \psi \in \mathscr{H},
$$

заведомо принадлежит $L_{1}^{\text {loc }}\left(\mathbb{R}_{+}\right)$по переменной $s$ и непрерывно по $t$. Последовательность вполне положительных отображений $P_{\tau, t}^{(n)}(\cdot)(n=0,1, \ldots)$,

$$
\begin{gathered}
P_{t, \tau}^{(0)}(B)=W_{t, \tau}^{*} B W_{t, \tau} \stackrel{\text { def }}{=} V_{t, \tau}(B) \\
P_{t, \tau}^{(n+1)}(B) \stackrel{\text { def }}{=} V_{t, \tau}(B)+\int_{\tau}^{t} d s V_{t, s} \Phi_{s} P_{s, \tau}^{(n)}(B) \\
=V_{t, \tau}(B)+\sum_{k=1}^{n+1} \int_{\tau}^{t} d s_{1} V_{t, s_{1}} \Phi_{s_{1}} \cdots \int_{\tau}^{s_{k-1}} d s_{k} V_{s_{k-1}, s_{k}} \Phi_{s_{k}} V_{s_{k}, \tau}(B)
\end{gathered}
$$

корректно определена как последовательность ограниченных операторов, соответствуюших последовательности квадратичных форм на $\operatorname{dom} \Lambda^{1 / 2}$, равномерно ограниченных в $\mathscr{H}$. Очевидно, что $P_{t, \tau}^{(0)}(B) \leqslant\|B\| \cdot I$, и с помощью $(1.2)$ по индукции получаем

$$
P_{t, \tau}^{(n+1)}(B) \leqslant\|B\|\left(V_{t, \tau}(I)+\int_{\tau}^{t} d s V_{t, s} \Phi_{s}(I)\right)=\|B\| \cdot I
$$

для любого эрмитова оператора $B$. Таким образом, последовательность вполне положительных отображений $P_{t, \tau}^{(n)}(\cdot)$ монотонно возрастает с увеличением $n$ и является равномерно ограниченной: $\left\|P_{t, \tau}^{(n)}(B)\right\| \leqslant\|B\|$. Поэтому существует наименьшая верхняя грань [8]

$$
P_{t, s}^{\min }(B)=\text { l.u.b. } P_{t, s}^{(n)}(B),
$$

которая в силу нормальности отображения $\Phi_{s}(\cdot)$ удовлетворяет интегральному уравнению 4

$$
P_{t, \tau}^{\min }=V_{t, \tau}(B)+\int_{\tau}^{t} d s V_{t, s} \Phi_{s} P_{s, \tau}^{\min }(B) .
$$

Заметим, что из (1.2), (1.3) следует, что

$$
\begin{aligned}
P_{t, \tau}^{(1)}(I) & =V_{t, \tau}(I)+\int_{\tau}^{t} d s V_{t, s} \Phi_{s} V_{s, \tau}(I) \\
& =V_{t, \tau}(I)+\int_{\tau}^{t} d s V_{t, s} \Phi_{s}(I)-\int_{\tau}^{t} d s_{1} V_{t, s_{1}} \Phi_{s_{1}} \int_{\tau}^{s_{1}} d s_{2} V_{s_{1}, s_{2}} \Phi_{s_{2}}(I) \\
& =I-\int_{\tau}^{t} d s_{1} V_{t, s_{1}} \Phi_{s_{1}} \int_{\tau}^{s_{1}} d s_{2} V_{s_{1}, s_{2}} \Phi_{s_{2}}(I) \stackrel{\text { def }}{=} I-\Delta_{t, \tau}^{(2)} .
\end{aligned}
$$

\footnotetext{
${ }^{4}$ В случае ограниченных коэффициентов $H$ и $\Phi(\cdot)$ это уравнение эквивалентно приведенному выше диффференциальному уравнению (1.1) и поэтому назьвается интегральным уравнением марковской эволюиии. Конструкция минимального решения аналогична конструкции минимального решения для уравнений с постоянньми коэффициентами [3], [4]; существование верхней грани и ее минимальные свойства подробно обсуждались в [4] и [6].
} 
Используя разложение (1.3) и последовательно применяя тождества (1.2), получаем

$$
\begin{gathered}
P_{t, \tau}^{(n)}(I)=I-\Delta^{(n+1)}(t, \tau) \\
\Delta_{t, \tau}^{(n+1)} \stackrel{\text { def }}{=} \int_{\tau}^{t} d s_{1} V_{t, s_{1}} \Phi_{s_{1}} \cdots \int_{\tau}^{s_{n}} d s_{n+1} V_{s_{n}, s_{n+1}} \Phi_{s_{n+1}}(I) \\
=\int_{\tau}^{t} d s V_{t, s} \Phi_{s}\left(\Delta^{(n)}(s, \tau)\right) \leqslant I
\end{gathered}
$$

причем последовательность $P_{t, \tau}^{(n)}(I)$ монотонно возрастает при увеличении $n$, а последовательность $\Delta_{t, s}^{(n)}$ монотонно убывает. Следовательно, $P_{t, \tau}^{(n)}(I) \uparrow I$ в сильном смысле при $n \rightarrow \infty$ тогда и только тогда, когда $\Delta_{t, s}^{(n)} \downarrow 0$ сходится слабо [8].

Чтобы получить более простое достаточное условие унитальности, рассмотрим оценки для интеграла от положительного оператора $P_{t, \tau}^{(n)} \Phi_{\tau}(I)$. Из (1.2) имеем

$$
\begin{aligned}
\int_{\tau}^{t} d s P_{t, s}^{(n)}\left(\Phi_{s}(I)\right)= & \int_{\tau}^{t} d s V_{t, s}\left(\Phi_{s}(I)\right)+\cdots \\
& +\int_{\tau}^{t} d s \int_{s}^{t} d s_{1} V_{t, s_{1}} \Phi_{s_{1}} \cdots \int_{s}^{s_{n-1}} d s_{n} V_{s_{n-1}, s_{n}} \Phi_{s_{n}} V_{s_{n}, s} \Phi_{s}(I)
\end{aligned}
$$

В последнем интеграле все переменные $s_{k}$ принимают значения большие $s$, т.е.область интегрирования имеет вид $\tau \leqslant s \leqslant s_{n} \leqslant \cdots \leqslant s_{1} \leqslant t$. Следовательно, меняя порядок интегрирования так, чтобы интегрирование по $s$ выполнялось в первую очередь, получаем

$$
\begin{aligned}
\int_{\tau}^{t} d s P_{t, s}^{(n)}\left(\Phi_{s}(I)\right)= & \int_{\tau}^{t} d s V_{t, s} \Phi_{s}(I)+\cdots \\
& +\int_{\tau}^{t} d s_{1} V_{t, s_{1}} \Phi_{s_{1}} \cdots \int_{\tau}^{s_{n-1}} d s_{n} V_{s_{n-1}, s_{n}} \Phi_{s_{n}} \int_{\tau}^{s_{n}} d s V_{s_{n}, s} \Phi_{s}(I) \\
\leqslant & n I .
\end{aligned}
$$

Последний интеграл в (1.5) совпадает с интегралом в (1.4) при замене $s \rightarrow s_{n+1}$. Сравнивая (1.5) и (1.4), мы получаем следующее важное равенство:

$$
C_{t, \tau}^{(n)}=\int_{\tau}^{t} d s P_{t, s}^{(n)}\left(\Phi_{s}(I)\right)=\sum_{k=1}^{n} \Delta_{t, \tau}^{(k)}
$$

Поэтому если существует плотно определенньй оператор такой, что $C_{t, \tau} \geqslant C_{t, \tau}^{(n)}$ сразу для всех $n$, то убывающая последовательность операторов $\Delta_{t, \tau}^{(n)}($ см. (1.4)) заведомо слабо сходится к 0 , а из равномерной ограниченности операторов $\Delta_{t, \tau}^{(n)}$, положительности и слабой сходимости следует их сильная сходимость. Таким образом, справедливо следующее утверждение. 
ТЕорема 1.1. При условиях (A), (В) минимальное решение уравнения марковской әволюиии (1.1) является унитальным на любом отрезке $T \subseteq[\tau, t]$, если существует плотно определенный оператор $C_{t, \tau}$ такой, что

$$
C_{t, \tau} \geqslant \int_{\tau}^{t} d s P_{t, s}^{(n)}\left(\Phi_{s}(I)\right)
$$

равномерно по п.

Грубо говоря, для консервативности минимального решения достаточно, чтобы оператор $\int_{\tau}^{t} d s P_{t, s}^{\min }\left(\Phi_{s}(I)\right)$ был плотно определен.

Пусть $a_{n} \geqslant 0, \sum_{n} a_{n}=\infty$. Поскольку последовательность $\Delta^{(n)}(t, \tau)$ положительна и убьвает монотонно, то сумма

$$
\Delta_{t, \tau}^{(n)} \stackrel{\text { def }}{=} \sum_{k=1}^{n} a_{k} \Delta_{t, \tau}^{(k)}
$$

может быть равномерно ограничена сверху плотно определенным оператором, только если $\Delta^{(n)}(t, \tau)$ слабо сходится к 0 . Ряд, соответствующий этой сумме с $a_{n}=n^{-1}$, определяется решением регуляризованного в смысле [2] уравнения с генератором $\mathscr{L}_{t}^{(\lambda)}(\cdot)$ :

$$
\mathscr{L}_{t}^{(\lambda)}(B)=\lambda \Phi_{t}(B)-G_{t}^{*} X-X G_{t}, \quad \lambda \in(0,1) .
$$

Более точно, сходящийся по норме ряд, представляющий решение уравнения

$$
\frac{\partial}{\partial t} T_{t, s}^{(\lambda)}(B)=\mathscr{L}_{t}^{(\lambda)}\left(T_{s, t}^{(\lambda)}(B)\right),\left.\quad T_{t, s}^{(\lambda)}(B)\right|_{s=t}=B
$$

имеет следующий вид [2]:

$$
T_{t, \tau}^{(\lambda)}(B)=V_{t, \tau}(B)+\sum_{n=1}^{\infty} \lambda^{n} \int_{\tau}^{t} d s_{1} V_{t, s_{1}} \Phi_{s_{1}} \cdots \int_{\tau}^{s_{n-1}} d s_{n} V_{s_{n-1}, s_{n}} \Phi_{s_{n}} V_{s_{n}, \tau}(B) .
$$

Из этого тождества и определения (1.4) следует неравенство

$$
\int_{(0,1)} d \lambda \int_{\tau}^{t} d s T_{t, s}^{(\lambda)}\left(\Phi_{s}(I)\right) \geqslant \sum_{n=1}^{N} \frac{1}{n} \Delta_{t, \tau}^{(n)} \quad \forall N>1 .
$$

Поэтому верно следующее утверждение.

ТЕорема 1.2. Предположим, что условия (A), (В) выполнены. Если оператор

$$
\widehat{C}_{t, \tau}=\int_{(0,1)} d \lambda \int_{\tau}^{t} d s T_{t, s}^{(\lambda)}\left(\Phi_{s}(I)\right)
$$

плотно определен в $\mathscr{H}$, то минимальная КДП унитальна на любом отрезке $T \subseteq[\tau, t]$

Поскольку $T_{t, s}^{(\lambda)}(\cdot) \leqslant P_{t, s}^{\min }(\cdot)$, то очевидно, что наличие плотной области определения оператора $C_{t, \tau}$ влечет за собой существование более широкой области определения оператора $\widehat{C}_{\tau, t}$. Рассмотрим априорные оценки для оператора $P_{t, s}\left(\Phi_{s}(I)\right)$ и сформулируем условия, при которых имеет место оценка

$$
\left(\psi, P_{t, s}(\Phi(I)) \psi\right) \leqslant\left(\psi, \Lambda e^{\int_{(s, t)} c(\tau) d \tau} \psi\right) \quad \forall \psi \in \operatorname{dom} \Lambda,
$$

для некоторого положительного самосопряженного оператора $\Lambda$ и $c(s) \in L_{1}^{\text {loc }}\left(\mathbb{R}_{+}\right)$. 
2. Априорные оценки. Пусть $\Lambda \geqslant \Phi_{t}(I) \geqslant 0$ и $\Lambda$ и $H_{\text {s.a. }}$ - самосопряженные операторы такие, что сумма $H_{\text {int }}=H_{\text {s.a. }}+\Lambda$-положительный самосопряженный оператор с существенной областью определения $\operatorname{dom} \Lambda$. Не уменьшая общности, будем считать, что оператор $\Lambda$ имеет ограниченный обратный. Обозначим через $\mathscr{H}_{k}$ шкалу гильбертовых пространств $\mathscr{H}_{k}$ со скалярньм произведением $(g, h)_{k}=\left(\Lambda^{k / 2} g, \Lambda^{k / 2} h\right)$ и через $\mathscr{T}_{k}$ шкалу пространств $\mathscr{T}_{k}=\left\{\sigma=\Lambda^{-k / 2} \rho \Lambda^{-k / 2}, \rho \in \mathscr{T}\right\}[6]$.

ЛЕмма 2.1. Если существуют положительные постоянные $\mu$ и $\nu$ такие, что

$$
0 \leqslant \delta \Lambda^{2} \leqslant H_{\text {int }}^{2} \leqslant \nu \Lambda^{2}
$$

то унитарная группа $U_{t}=e^{i t H_{\mathrm{int}}}$ равномерно ограничена и сильно непрерывна в гильбертовых пространствах $\mathscr{H}_{k}(k=0,1,2)$.

ДокАЗАТЕЛЬСтво. Сильная непрерьвность в $\mathscr{H}=\mathscr{H}_{0}$ следует из самосопряженности оператора $H_{\text {int }}$.

Поскольку из неравенства $A \geqslant B \geqslant 0$ следует $A^{\alpha} \geqslant B^{\alpha} \geqslant 0$ для любого $\alpha \in(0,1)$, TO

$$
\Lambda_{t}^{k}=U_{t}^{*} \Lambda^{k} U_{t} \leqslant \delta^{-k / 2} U_{t}^{*} H_{\mathrm{int}}^{k} U_{t}=\delta^{-k / 2} H_{\mathrm{int}}^{k} \leqslant c^{k / 2} \Lambda^{k},
$$

где $c=\nu / \delta$. Из полученного вьше неравенства имеем

$$
\left\|\Lambda^{k / 2} U_{t} \psi\right\|^{2}=\left\|U_{t}^{*} \Lambda^{k / 2} U_{t} \psi\right\|^{2}=\left\|\left(U_{t}^{*} \Lambda U_{t}\right)^{k / 2} \psi\right\|^{2} \leqslant c^{k / 2}\left\|\Lambda^{k / 2} \psi\right\|^{2} .
$$

С другой стороны, поскольку $U_{t}=e^{i t H_{\text {int }}}$ и $H_{\text {int }}$ коммутируют, то из неравенства $(2.1)$ имеем

$$
\left\|\Lambda^{k / 2}\left(U_{t}-U_{s}\right) \psi\right\|^{2} \leqslant \delta^{-k / 2}\left\|H_{\mathrm{int}}^{k / 2}\left(U_{t}-U_{s}\right) \psi\right\|^{2}=\left\|\left(U_{t}-U_{s}\right) H_{\mathrm{int}}^{k / 2} \psi\right\|^{2} \rightarrow 0
$$

при $t-s \rightarrow 0$, поскольку $H_{\mathrm{int}}^{k / 2} \psi \in \mathscr{H}$ для любого $\psi \in \mathscr{H}_{k}$.

ЛЕМма 2.2. Пусть $-G_{0}$ - генератор сильно непрерывной сжимающей полугрупnьь $W_{t}=e^{-t G_{0}}$ в $\mathscr{H}$. Если существует постоянная $c>0$ такая, что

$$
-2 \operatorname{Re}\left(\Lambda^{k / 2} G_{0} \psi, \Lambda^{k / 2} \psi\right) \leqslant c\left\|\Lambda^{k / 2} \psi\right\|^{2}
$$

для любого $\psi \in \mathscr{H}_{k}(k=1,2)$, то полугруппа $W_{t}$ ограничена и сильно непрерывна в $\mathscr{H}_{k}$.

ДокАЗАТЕЛЬСТво. Пусть $\psi$-решение резольвентного уравнения $\left(\lambda G_{0}+I\right) \psi=\varphi$ и $\varphi \in \mathscr{H}_{k}$, т.е. $\varphi=\Lambda^{-k / 2} \theta, \theta \in \mathscr{H}$. Тогда $\left\|\Lambda^{k / 2}\left(\lambda G_{0}+I\right) \psi\right\|^{2}=\|\theta\|^{2}$ и в силу оценки $(2.2)$ имеем $2 \operatorname{Re}\left(\Lambda^{k / 2} G_{0} \psi, \Lambda^{k / 2} \psi\right) \geqslant-c\left\|\Lambda^{k / 2} \psi\right\|^{2}$. Поэтому при всех $\lambda>2 c$ имеем

$\|\theta\|^{2}=\left\|\Lambda^{k / 2}\left(\lambda G_{0}+I\right) \psi\right\|^{2} \geqslant(1-2 \lambda c)\left\|\Lambda^{k / 2} \psi\right\|^{2}=(1-2 \lambda c)\left\|\Lambda^{k / 2}\left(\lambda G_{0}+I\right)^{-1} \Lambda^{-k / 2} \theta\right\|^{2}$.

Следовательно, $\left\|\left(I+G_{0} t / N\right)^{-1}\right\|_{k}=\left\|\left(I+G_{k} t / N\right)^{-1}\right\| \leqslant(1-2 c t / N)^{-1 / 2}$, где $G_{k}=$ $\Lambda^{k / 2} G_{0} \Lambda^{-k / 2}$. Учитьвая, что

$$
W_{t}=s-\lim _{N \rightarrow \infty}\left(I+\frac{G_{0} t}{N}\right)^{-N},
$$


получаем

$$
\begin{aligned}
\left\|\Lambda^{k / 2} W_{t} \Lambda^{-k / 2}\right\| & =\lim _{N \rightarrow \infty}\left\|\left(I+\frac{G_{k} t}{N}\right)^{-N}\right\| \leqslant\left\|\left(I+\frac{G_{k} t}{N}\right)\right\|^{N} \\
& \leqslant \lim _{N \rightarrow \infty}\left(1-\frac{2 c t}{N}\right)^{-N / 2}=e^{c t},
\end{aligned}
$$

что доказьвает ограниченность полугрупшы $W_{t}$ в $\mathscr{H}_{k}$. Попутно можно заметить, что оценка нормы резольвентного оператора $\left(I+\lambda G_{0}\right)^{-1}$ в $\mathscr{H}_{k}$ совпадает с оценкой нормы резольвентного оператора $\left(I+\lambda G_{k}\right)^{-1}$ в $\mathscr{H}$.

Нетрудно видеть, что поскольку оператор $\Lambda$ замкнут и ограниченно обратим, а оператор $G_{0} \Lambda^{-k / 2}$ замкнут, то $G_{k}$ также замкнут в $\mathscr{H}$ (см. [10, гл. III, $\S 5$, п. 2, задача 5.12]). Таким образом, оператор $-G_{k}$ удовлетворяет условиям теоремы о генераторе квазиограниченной сильно непрерьвной полугрупшы $W_{t}^{(k)}=\Lambda^{k / 2} W_{t} \Lambda^{-k / 2}=e^{-t \Lambda^{k / 2} G_{0} \Lambda^{-k / 2}}$, действующей в $\mathscr{H}$ (см. [10, гл. IX, $§ 1$, п. 4]).

Поскольку полугруппа $W_{t}$ ограничена в $\mathscr{H}_{k}$, то достаточно доказать ее непрерьвность наплотном подмножестве $\mathscr{H}_{\mu, k}=\Lambda^{k / 2}\left(\mu G_{0}+I\right)^{-N} \Lambda^{-k / 2} \mathscr{H} \subset \mathscr{H}$. Пусть $h \in \mathscr{H}$ и $\psi=\Lambda^{k / 2}\left(\mu G_{0}+I\right)^{-N} \Lambda^{-k / 2} h \in \mathscr{H}_{\mu, k}$. Тогда $\left\|\Lambda^{k / 2}\left(W_{t}-I\right) \Lambda^{-k / 2} \psi\right\|=\left\|\left(W_{t}^{(k)}-I\right) \psi\right\|$. Теперь сильная непрерывность $W_{t}$ в $\mathscr{H}_{k}$ следует из сильной непрерьвности $W_{t}^{(k)}$ в $\mathscr{H}$.

Комбинируя две предыдущие леммы, мы получаем следующий результат.

Лемма 2.3. Предположим, что условия (2.1), (2.2) выполнены. Тогда

(i) коиикл $W_{t, s}=U_{t}^{*} W_{t-s} U_{s}$ сильно непрерывен $и$ ограничен в $\mathscr{H}_{k}, k=1,2$;

(ii) при условии

$$
A_{k, n}=\Lambda^{(k-1) / 2} \Phi_{n} \Lambda^{-k / 2} \in \mathscr{B}(\mathscr{H}), \quad \sum_{n} A_{k, n}^{*} A_{k, n} \in \mathscr{B}(\mathscr{H}), \quad k=1,2,
$$

квадратичная форма $f_{t, s}=\Phi_{t, *}\left(P_{t}\right)\left[W_{t, s} \psi\right], \psi \in \mathscr{H}_{k}$, ограничена и непрерывна по $t, s$ для любого семейства операторов $P_{t}$, ограниченного и сильно непрерывного в $\mathscr{H}_{k-1}$.

ДокАЗАТЕльство. Первое утверждение леммы есть очевидное следствие ограниченности и сильной непрерывности полугрупп $U_{t}$ и $W_{t}$ в $\mathscr{H}_{k}$.

Учитывая, что $\Phi_{t}(X)=\sum_{n} U_{t}^{*} \Phi_{n}^{*} U_{t} X U_{t}^{*} \Phi_{n} U_{t}$ и $W_{t, s}=U_{t}^{*} W_{t-s} U_{s}$, запишем квадратичную форму $f_{t, s}$ как сумму $\sum_{n}\left(h_{k}(t, s), B_{k, n}(t) h_{k}(t, s)\right)$, где

$$
\begin{gathered}
h_{k}(t, s)=W_{t-s}^{(k)} U_{s}^{(k)} h, \quad U_{t}^{(k)}=\Lambda^{k / 2} U_{t} \Lambda^{-k / 2}, \quad W_{t}^{(k)}=\Lambda^{k / 2} W_{t} \Lambda^{-k / 2}, \\
\psi=\Lambda^{-k / 2} h, \quad h \in \mathscr{H}, \quad A_{k, n}=\Lambda^{(k-1) / 2} \Phi_{n} \Lambda^{-k / 2} \in \mathscr{B}(\mathscr{H}), \\
B_{k, n}(t)=A_{k, n}^{*} U_{t}^{(k-1)} \Lambda^{-(k-1) / 2} P_{t} \Lambda^{-(k-1) / 2} U_{-t}^{(k-1)} A_{k, n} \in \mathscr{B}(\mathscr{H}) .
\end{gathered}
$$

Поскольку операторы $U_{t}^{(k)}, W_{t-s}^{(k)}$ ограничены и сильно непрерывны в $\mathscr{H}$, то векторы $h_{k}(t, s)$ непрерывны по норме $\mathscr{H}$ по переменным $t$ и $s$ и в сумме

$$
\begin{aligned}
\left|f_{t, s}-f_{\tau, s}\right| \leqslant & \sum_{n}\left\{\left(\left\|h_{k}(t, s)\right\|+\left\|h_{k}(\tau, s)\right\|\right) \cdot\left\|B_{k, n}(s)\right\| \cdot\left\|h_{k}(t, s)-h_{k}(\tau, s)\right\|\right. \\
& \left.+\left\|h_{k}(t, s)\right\| \cdot\left\|\left(B_{k, n}(t)-B_{k, n}(\tau)\right) h_{k}(t, s)\right\|\right\}
\end{aligned}
$$


каждое слагаемое сходится к нулю при $\tau \uparrow t$. Заметим, что

$$
f_{k}(t, s) \leqslant\left\|B_{t}\right\| \sum_{n}\left(W_{t-s}^{(k)} U_{s}^{(k)} h, A_{k, n}^{*} A_{k, n} W_{t-s}^{(k)} U_{s}^{(k)} h\right)
$$

где по предположению ряд $\sum_{n} A_{k, n}^{*} A_{k, n}$ сильно сходится к ограниченному оператору. Сильная сходимость равномерна на компактных множествах, а множество векторов $\left\{W_{t-s}^{(k)} U_{s}^{(k)} h, s \leqslant t, t \in T\right\}$ при фиксированном $h$ компактно для любого компакта $T \in \mathbb{R}_{+}$. Поэтому из теоремы Лебега следует, что в сумме возможен почленный предельный переход.

В частности, при $k=1$ выполнены предположения $(\mathbf{A})$ и $(\mathbf{B})$, за исключением предположения о симметричности оператора $H$ на $\operatorname{dom} \Lambda^{1 / 2}$. Это условие можно заменить предположением о симметричности $H$ на $\operatorname{dom} \Lambda$, если коцикл $W_{t, s}$ оставляет инвариантным это множество. Предположим, что для любого $\psi \in \operatorname{dom} \Lambda$ выполнено

(C) условие относительной ограниченности (ср. [5] и [7]):

$$
\sum_{k}\left\|\Lambda^{1 / 2} \Phi_{k, t} \psi\right\|^{2}-2 \operatorname{Re}\left(\Lambda^{1 / 2} G_{t} \psi, \Lambda^{1 / 2} \psi\right) \leqslant c_{t}\left\|\Lambda^{1 / 2} \psi\right\|, \quad c_{t} \in L_{1}^{\text {loc }}\left(\mathbb{R}_{+}\right), \quad c_{t} \geqslant 0
$$

ЛЕмма 2.4. Если выполнены условия (2.1)-(2.3) для $k=2$, то условие (C) обеспечивает априорную оченку (1.7) в слабой форме.

ДокАЗАТЕЛЬСтво. Коцикл $W_{t, s}$ оставляет инвариантньми пространства $\mathscr{H}_{k}, k=$ 1,2 , поэтому оператор $V_{t, s}(\Lambda)$ корректно определен как оператор, соответствующий замыканию положительно определенной квадратичной формы $\left\|\Lambda^{1 / 2} W_{t, s} \psi\right\|^{2}$ на $\mathscr{H}_{2}$. По этой же причине очевидно, что для всех $0 \leqslant \tau \leqslant t$ из неравенства $(\mathbf{C})$ следует оценка

$$
\frac{\partial}{\partial t}\left\|\Lambda^{1 / 2} W_{t, \tau} \psi\right\|^{2}=-2 \operatorname{Re}\left(\Lambda^{1 / 2} G_{t} W_{t, \tau} \psi, \Lambda^{1 / 2} W_{t, \tau} \psi\right) \leqslant c_{t}\left\|\Lambda^{1 / 2} W_{t, \tau} \psi\right\|^{2}
$$

Решая задачу Коши для приведенного вьше дифференциального неравенства, мы получаем оценку

$$
V_{t, \tau}(\Lambda)_{*}[\psi] \leqslant e^{\int_{\tau}^{t} d s c_{s}}(\Lambda)_{*}[\psi]
$$

Следовательно, оператор $P_{t, \tau}^{(0)}(\Lambda)$, отвечающий квадратичной форме $\left\|\Lambda^{1 / 2} W_{t, s} \psi\right\|^{2}$, удовлетворяет неравенству

$$
P_{t, \tau}^{(0)}(\Lambda)=W_{t, \tau}^{*} \Lambda W_{t, \tau}=V_{t, \tau}(\Lambda) \leqslant \Lambda e^{\int_{\tau}^{t} d s c_{s}}
$$

Предположим, что

$$
P_{t, \tau}^{(n)}(\Lambda)_{*}[\psi] \leqslant e^{\int_{\tau}^{t} d s c_{s}}\left\|\Lambda^{1 / 2} \psi\right\|^{2}
$$


на $\operatorname{dom} \Lambda$, и докажем аналогичное неравенство для $P_{t, \tau}^{(n+1)}(\Lambda)$ по индукции. Из определения $P_{t, \tau}^{(n+1)}(\Lambda)$ и предположения $(\mathbf{C})$ имеем

$$
\begin{aligned}
& P_{t, \tau}^{(n+1)}(\Lambda)_{*}[\psi] \stackrel{\text { def }}{=} V_{t, \tau}(\Lambda)_{*}[\psi]+\sum_{k} \int_{\tau}^{t} d s \sum_{k} P_{s, \tau}^{(n)}(\Lambda)_{*}\left[\Phi_{k, s} W_{t, s} \psi\right] \\
& \leqslant V_{t, \tau}(\Lambda)_{*}[\psi]+\int_{\tau}^{t} d s e^{\int_{\tau}^{s} d r c_{r}} \sum_{k} \Lambda_{*}\left[\Phi_{k, s} W_{t, s} \psi\right] \\
& =\Lambda_{*}\left[W_{t, \tau} \psi\right]+\int_{\tau}^{t} d s e^{\int_{\tau}^{s} d r c_{r} \sum_{k}\left\|\Lambda^{1 / 2} \Phi_{k, s} W_{t, s} \psi\right\|^{2}} \\
& \leqslant \Lambda_{*}\left[W_{t, \tau} \psi\right]+\int_{\tau}^{t} d s e^{\int_{\tau}^{s} d r c_{r}}\left(c_{s} \Lambda_{*}\left[W_{t, s} \psi\right]+2 \operatorname{Re}\left(\Lambda^{1 / 2} G_{s} W_{t, s} \psi, \Lambda^{1 / 2} W_{t, s} \psi\right)\right) \\
& =\Lambda_{*}\left[W_{t, \tau} \psi\right]+\int_{\tau}^{t} d s \frac{\partial}{\partial s}\left(e^{\int_{\tau}^{s} d r c_{r}}\left\|\Lambda^{1 / 2} W_{t, s} \psi\right\|^{2}\right)=\left\|\Lambda^{1 / 2} \psi\right\|^{2} e^{\int_{\tau}^{t} d s c_{s}},
\end{aligned}
$$

где все неравенства понимаются в смысле неравенств для квадратичных форм, корректно определенных на $\operatorname{dom} \Lambda$, непрерьвно зависящих от $s$ и равномерно ограниченных в $\mathscr{H}_{1}$. Отсюда следует оценка (1.7).

Из оценки (1.7) следует, что операторы ограничены сверху плотно определенным оператором равномерно по $n$ :

$$
P_{t, \tau}^{(n)}\left(\Phi_{\tau}(I)\right) \leqslant P_{t, \tau}^{(n)}\left(\Lambda_{\tau}\right) \leqslant \Lambda e^{\int_{\tau}^{t} d s c_{s}},
$$

и, следовательно, достаточное условие унитальности из теорем 1.1 и 1.2 выполнено. Кроме того, для двойственного коцикла $T_{t, \tau}(\cdot)$, действуюшего в алгебре операторов с конечным следом $\mathscr{T}(\mathscr{H})$, вьполнена оценка

$$
\operatorname{Tr}\left\{B T_{t, \tau}(\rho)\right\}=\operatorname{Tr}\left\{P_{t, \tau}(B) \rho\right\} \leqslant e^{\int_{\tau}^{t} d s c_{s}}\left\|\Lambda^{-1 / 2} B \Lambda^{-1 / 2}\right\| \operatorname{Tr}\left\{\Lambda^{1 / 2} \rho \Lambda^{1 / 2}\right\} .
$$

Поэтому справедливо следующее утверждение.

ТЕОрема 2.1. Пусть выполнены условия (2.1)-(2.4). Тогда минимальная КДП унитальна на любом ограниченном отрезке $T \in \mathbb{R}_{+}$. Двойственная полугруппа сохраняет след начального состояния и оставляет инвариантной алгебру состояний $\mathscr{T}_{1}=\mathscr{T}\left(\mathscr{H}_{1}\right)$, порождаемую линейной оболочкой чистых состояний $\rho=|\psi\rangle\langle\psi|$, $\psi \in \mathscr{H}_{1},\|\rho\|_{1}=\operatorname{Tr}\left\{\Lambda^{1 / 2} \rho \Lambda^{1 / 2}\right\}$.

Рассмотрим условия, при которых предположение $(\mathbf{C})$ для генератора $\mathscr{L}_{t}(\cdot)$ с переменными коэффициентами может быть заменено на аналогичное условие для генератора $\mathscr{L}_{0}(\cdot)$ с постоянными коэффициентами.

Лемма 2.5. Предположим, что для формального генератора с постоянными коэффициентами $\mathscr{L}_{0}(\cdot)=\mathscr{L}(\cdot)-i\left[H_{\mathrm{int}}, \cdot\right]$ выполнена оченка $\mathscr{L}_{0}(\Lambda)_{*}[\psi] \leqslant c_{0}\left\|\Lambda^{1 / 2} \psi\right\|^{2}$ $\forall \psi \in \operatorname{dom} \Lambda$ и существуют полохительные постоянные $\delta, \mu, \nu$ и показатель степени $\varepsilon \in(0,1)$ такие, что на $\operatorname{dom} \Lambda$ в том жсе смысле справедливы неравенства

$$
-H_{\text {s.a. }} \leqslant \mu H_{\text {int }}^{\varepsilon}, \quad \Phi\left(H_{\text {s.a. }}\right) \leqslant c_{1} \Lambda, \quad \Phi\left(H_{\text {int }}^{\varepsilon}\right) \leqslant c_{2} \Lambda .
$$

Тогда для генератора $\mathscr{L}_{t}(\cdot): \mathscr{L}_{t}(B)=U_{t}^{*} \mathscr{L}_{0}\left(U_{t} B U_{t}^{*}\right) U_{t}^{*}$ условие $(\mathbf{C})$ выполнено с постоянной $c=\frac{\nu}{\delta}\left(c_{0}+c_{1}+\mu c_{2}\right)$. 
ДокаЗАТЕЛЬСТво. Учитьвая, что $H_{\text {int }}=H_{\text {s.a. }}+\Lambda$ и вьполнены оценки $(2.4)$, получаем

$$
\begin{aligned}
\mathscr{L}_{t}(\Lambda) & =U_{t}^{*}\left(\Phi\left(U_{t} \Lambda U_{t}^{*}\right)-G_{0}^{*} \Lambda-\Lambda G_{0}\right) U_{t} \\
& =U_{t}^{*}\left(\Phi\left(U_{t}\left(H_{\mathrm{int}}-H_{\text {s.a. }}\right) U_{t}^{*}\right)-G_{0}^{*} \Lambda-\Lambda G_{0}\right) U_{t} \\
& =U_{t}^{*}\left(\Phi\left(H_{\text {s.a. }}-U_{t} H_{\text {s.a. }} U_{t}^{*}\right)+\Phi(\Lambda)-G_{0}^{*} \Lambda-\Lambda G_{0}\right) U_{t} \\
& \leqslant U_{t}^{*}\left(\Phi\left(H_{\text {s.a. }}-U_{t} H_{\text {s.a. }} U_{t}^{*}\right)+c_{0} \Lambda\right) U_{t} \leqslant U_{t}^{*}\left(\Phi\left(H_{\text {s.a }}\right)+\mu \Phi\left(H_{\text {int }}^{\varepsilon}\right)+c_{0} \Lambda\right) U_{t} \\
& \leqslant\left(c_{0}+c_{1}+\mu c_{2}\right) U_{t}^{*} \Lambda U_{t} \leqslant \frac{\nu}{\delta}\left(c_{0}+c_{1}+\mu c_{2}\right) \Lambda .
\end{aligned}
$$

Таким образом, если выполнены условия (2.1)-(2.4), то

$$
\mathscr{L}_{t}(\Lambda) \leqslant \lambda \Lambda, \quad \lambda=\frac{\nu}{\delta}\left(c_{0}+c_{1}+\mu c_{2}\right)
$$

и из теоремы 2.1 следует оценка $(1.7) ;$ поэтому генератор $\mathscr{L}_{t}(B) \stackrel{\text { def }}{=} U_{t}^{*} \mathscr{L}_{0}\left(U_{t} B U_{t}^{*}\right) U_{t}$ регулярен. С другой стороны, очевидно, что если $P_{t}(B)=e^{t \mathscr{L}}(B)$, где

$$
\mathscr{L}(\cdot)=\mathscr{L}_{0}(\cdot)+i\left[H_{\mathrm{int}}, \cdot\right]
$$

то формальный генератор коцикла $P_{s, t}(B)=U_{t}^{*} P_{t-s}\left(U_{s} B U_{s}^{*}\right) U_{t}$, унитарно эквивалентного $P_{t}(\cdot)$, совпадает с $\mathscr{L}_{t}(\cdot)$.

ТеОрема 2.2. Если условия (2.1)-(2.4) выполнены для $\mathscr{L}_{0}(\cdot)$ и $\mathscr{L}_{0}(\Lambda) \leqslant c_{0} \Lambda$, то генератор $\mathscr{L}(\cdot)=\mathscr{L}_{0}(\cdot)+i\left[H_{\mathrm{int}}, \cdot\right]$ регулярен.

В этом случае $\Lambda$-парой для генератора $\mathscr{L}(\cdot)$ мы называем пару $\left(\Lambda, H_{\text {int }}\right)$.

3. Структура генераторов в квантовой оптике. Типичньй формальньй генератор $\mathscr{L}(\cdot)$ уравнения марковской эволюции в квантовьй оптике (см. [11], [12]) действует в алгебре наблюдаемых $\mathscr{B}(\mathscr{H}), \mathscr{H}=\left(\ell_{2}\right)^{\otimes K} \otimes \mathbb{C}^{M}$, и имеет линдбладовскую форму $\mathscr{L}(B)=\Phi(B)-G^{*} B-B G$, где

$$
\Phi(B)=\sum_{k=1}^{K} \Phi_{k}(B), \quad G=\frac{1}{2} \Phi(I)+i H, \quad \Phi_{l}(B)=\lambda_{l} a_{l}^{\dagger} B a_{l} \quad \text { или } \Phi_{j}(B)=\mu_{j} a_{j} B a_{j}^{\dagger},
$$

a $\lambda_{l}, \mu_{j} \geqslant 0$ являются положительными операторами в $\mathbb{C}^{M}, a_{k}$ и $a_{k}^{\dagger}$ являются операторами рождения и уничтожения, действующими в $k$-м сомножителе тензорного произведения $\left(\ell_{2}\right)^{\otimes K}$, т.е. $\left[a_{k}, a_{n}\right]=0,\left[a_{n}, a_{k}^{\dagger}\right]=\delta_{k, n}$, и гамильтониан

$$
H=\sum_{j=1}^{J}\left(h_{j} \prod_{k=1}^{K}\left(a_{k}^{\dagger}\right)^{n_{j k}}\left(a_{k}\right)^{m_{j k}}+h_{j}^{*} \prod_{k=1}^{K}\left(a_{k}^{\dagger}\right)^{m_{j k}}\left(a_{k}\right)^{n_{j k}}\right)
$$

является симметричньм полиномом конечной степени

$$
\operatorname{deg} H=\max _{j} \sum_{k}\left(m_{j k}+n_{j k}\right) \leqslant 2 K \max _{j}\left\{m_{j k}, n_{j k}\right\}
$$


от операторов рождения и уничтожения с матричными коэффициентами $h_{j} \in \mathbb{C}^{M} \otimes \mathbb{C}^{M}$, действующим в $\mathscr{H}$ (см. [13], [14]).

Для любого оператора $\mathscr{H}$, представимого в виде полинома (3.2), существует диагональный оператор

$$
\Lambda=c_{\Lambda}\left(I+\sum_{k=1}^{K}\left(a_{k}^{\dagger} a_{k}\right)^{m_{k}}\right), \quad c_{\Lambda}>0
$$

такой, что $H$ ограничен относительно оператора $\Lambda$ с относительной верхней границей $O\left(c_{\Lambda}^{-1}\right)$. Действительно, $0 \leqslant\left(a^{\dagger}\right)^{2} a^{2}=\left(a^{\dagger} a\right)^{2}-a^{\dagger} a \leqslant\left(a^{\dagger} a\right)^{2}$. Поэтому $\left(a^{\dagger}\right)^{n} a^{n} \leqslant\left(a^{\dagger} a\right)^{n}$. $\mathrm{C}$ другой стороны, в силу неравенства $A^{*} B+B^{*} A \leqslant A^{*} A+B^{*} B$ имеем

$$
\begin{aligned}
& h_{j} \prod_{k=1}^{K}\left(a_{k}^{\dagger}\right)^{n_{j k}}\left(a_{k}\right)^{m_{j k}}+h_{j}^{*} \prod_{k=1}^{K}\left(a_{k}^{\dagger}\right)^{m_{j k}}\left(a_{k}\right)^{n_{j k}} \\
& \quad \leqslant\left|h_{j}\right|\left(\prod_{k=1}^{K}\left(a_{k}^{\dagger}\right)^{n_{j k}}\left(a_{k}\right)^{n_{j k}}+\prod_{k=1}^{K}\left(a_{k}^{\dagger}\right)^{m_{j k}}\left(a_{k}\right)^{m_{j k}}\right) \\
& \quad \leqslant\left|h_{j}\right|\left(\prod_{k=1}^{K} N_{k}^{n_{j k}}+\prod_{k=1}^{K} N_{k}^{m_{j k}}\right)
\end{aligned}
$$

где $N_{k}=a_{k}^{\dagger} a_{k}$ - коммутирующие между собой операторы числа частищ. Поэтому из неравенства

$$
\prod_{k=1}^{K} c_{k} \leqslant K^{-1} \sum_{k=1}^{K} c_{k}^{K}
$$

для положительных коммутируюших величин получаем оценку для оператора $H$ :

$$
H \leqslant \frac{J}{K} \max _{j}\left\{\left|h_{j}\right|\right\}\left(I+\sum_{k=1}^{K}\left(a_{k}^{\dagger} a_{k}\right)^{K \max _{j}\left\{m_{j k}, n_{j k}\right\}}\right) .
$$

ТЕОРема 3.1. Если гамильтониан $H$ имеет вид $H=H_{s}+H_{\mathrm{S} . \text { a. }}$, әде $H_{\mathrm{S} . \mathrm{a}}$ является самосопряженным полиномом конечной степени $M$ по операторам рохсдения и уничтожения $и H_{s}=H_{s}\left(a^{\dagger}, a\right)$ является эрмитовым оператором не выше второго порядка, то существуют $c_{\Lambda}>0 u m_{k} \geqslant M$ такие, что $\left\{\Lambda, H_{\text {int }}=H_{\text {s.a. }}+\Lambda\right\}$ является $\Lambda$-парой для генератора $\mathscr{L}(\cdot)(3.1),(3.2)$ и генератор $\mathscr{L}(\cdot)=\mathscr{L}_{0}(\cdot)+$ $i\left[H_{\mathrm{int}}, \cdot\right]$ регулярен.

ДокАЗАТЕЛЬСТво. Выбирая

$$
c_{\Lambda}>2\left(\max _{k}\left\{\lambda_{k}, \mu_{k}\right\}+\frac{J}{K} \max _{j}\left\{\left|h_{j}\right|\right\}\right), \quad m_{k} \geqslant 2 \max _{j}\left\{m_{j k}, n_{j k}\right\},
$$

в формуле (3.3), мы обеспечиваем ограниченность операторов $H$ и $\Phi(I)$ относительно оператора $\Lambda$ с относительной границей меньше $1 / 2$. Следовательно, для достаточно больших $c_{\Lambda}$ и $\mu=\min \left\{m_{k}\right\} \geqslant M$ согласно [10,гл. IX,$\S 2$, п. 3, задача 2.9] $H_{\text {int }}=H_{\text {s.a. }}+\Lambda$ является положительным самосопряженньм оператором таким, что $\operatorname{dom} H_{\text {int }}=\operatorname{dom} \Lambda$, a $G_{0}=i\left(H_{s}-\Lambda\right)+\Phi(I) / 2$ является аккреативным оператором [10, гл. V, $\S 3$, п. 10], 


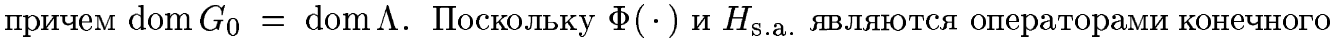
порядка, свойства $\Lambda$-пары (2.1)-(2.4) можно легко удовлетворить, выбирая $\mu$ и $c_{\Lambda}$ достаточно большими.

Коммутатор полинома второго порядка по операторам рождения и уничтожения с произвольным полиномом прядка $M<\infty$ имеет порядок $M$ или меньше. Следовательно, коммутатор $i\left[H_{0}, \Lambda\right]=i\left[H_{s}-\Lambda, \Lambda\right]=i\left[H_{s}, \Lambda\right], H_{0}=H_{s}-\Lambda$, является оператором того же или меньшего порядка, что и $\Lambda$, и, следовательно, существует постоянная $c \in \mathbb{R}$ такая, что $i\left[H_{0}, \Lambda\right] \leqslant c \Lambda$. Например, из формул коммутации $f\left(a^{\dagger} a\right) a^{\dagger}=a^{\dagger} f\left(a^{\dagger} a+I\right)$ и $f\left(a^{\dagger} a\right) a=a f\left(a^{\dagger} a-I\right)$ вытекают оценки

$$
\begin{aligned}
i\left[\left(a^{\dagger} a\right)^{m}, \lambda a^{\dagger}+\bar{\lambda} a\right] & \leqslant 2|\lambda| \sum_{k=1}^{m} C_{m}^{k}\left(a^{\dagger} a\right)^{k-1 / 2} \leqslant 2^{m+1}|\lambda|\left(a^{\dagger} a\right)^{m-1 / 2} \\
& \leqslant 2^{m+1}|\lambda| c_{\Lambda}{ }^{-1} \Lambda, \\
i\left[\left(a^{\dagger} a\right)^{m}, \lambda\left(a^{\dagger}\right)^{2}+\bar{\lambda} a^{2}\right] & \leqslant 2|\lambda| \sum_{k=1}^{m} 2^{m-k} C_{m}^{k}\left(a^{\dagger} a\right)^{k} \leqslant 2^{2 m+1}|\lambda|\left(a^{\dagger} a\right)^{m} \\
& \leqslant 2^{2 m+1}|\lambda| c_{\Lambda}{ }^{-1} \Lambda .
\end{aligned}
$$

Аналогичные неравенства справедливы для коммутаторов с $\Lambda^{2}$.

Простые алгебраические вычисления показьвают, что для вполне положительного отображения (3.1) оператор $\Phi(\Lambda)-(\Lambda \Phi(I)+\Phi(I) \Lambda) / 2$ является полиномом того же порядка, что и $\Lambda$, а оператор $\Phi\left(\Lambda^{2}\right)-\left(\Lambda^{2} \Phi(I)+\Phi(I) \Lambda^{2}\right) / 2$ имеет тот же порядок, что и $\Lambda^{2}$. Например, для любого $l \geqslant 0$ имеет место следующая оценка: $\left(a_{k}^{\dagger}\right)^{l} \Lambda a_{k}^{l}-\left(\left(a_{k}^{\dagger}\right)^{l} a_{k}^{l}\right) \circ \Lambda \leqslant 0$; с другой стороны, существует постоянная $c \geqslant 0$ такая, что $a_{k} \Lambda a_{k}^{\dagger}-\left(a_{k} a_{k}^{\dagger}\right) \circ \Lambda \leqslant c \Lambda$ для оператора $\Lambda$ (3.3). Поэтому условие $\mathscr{L}_{0}\left(\Lambda^{k}\right) \leqslant c_{k} \Lambda^{k}$ выполнено для $\Lambda$ и $\Lambda^{2}$. Следовательно, выполнено и более слабое условие (2.2). Конструкция операторов $\Lambda$ и $H_{\mathrm{int}}$ обеспечивает выполнение условий $(2.1)$ и (2.3), (2.4). Нетрудно видеть, что эрмитовы операторы второго порядка симметричны на $\operatorname{dom} \Lambda$. Следовательно, вьполнены условия теоремы 2.2. Теорема доказана.

4. Примеры. В этом пункте мы рассмотрим класс гамильтонианов и вполне положительных отображений, для которых выполнены условия теоремы 3.1, которая практически сводится к проверке самосопряженности главной части оператора $H$.

1. Пусть $\lambda$ является комплексным числом и $m, n \geqslant 0$. Пусть

$$
H=\lambda\left(a_{1}^{\dagger}\right)^{m} a_{2}^{n}+\bar{\lambda} a_{1}^{m}\left(a_{2}^{\dagger}\right)^{n}, \quad \lambda \in \mathbb{C} .
$$

Докажем, что все гамильтонианы такого вида существенно самосопряжены в $\mathscr{H}_{2}=$ $\ell_{2} \otimes \ell_{2}$. Достаточно доказать, что не существует вектора

$$
\psi=\left\{\psi_{k, j}, k, j \geqslant 1, \sum_{k, j}\left|\psi_{k, j}\right|^{2}=\|\psi\|_{\mathscr{H}_{2}}^{2}\right\}
$$

такого, что $H \psi= \pm i \psi[15]$. Мы полагаем $\psi_{k, j}=0$, если $\min \{k, j\} \leqslant 0$.

Запишем уравнения для компонент вектора $\psi_{k, j}$ следующим образом:

$$
\pm i \psi_{k, j}=\lambda A_{k, j}^{m, n} \psi_{k-m, j+n}+\bar{\lambda} B_{k, j}^{m, n} \psi_{k+m, j-n}, \quad A_{k, j}^{m, n}, B_{k, j}^{m, n} \geqslant 0 .
$$


Положим $\psi_{k, j}=0$, если $\min \{k, j\} \leqslant 0$, и опустим точные выражения для функций $A_{k, j}^{m, n}$ и $B_{k, j}^{m, n}$, поскольку они не играют роли в доказательстве. Важное свойство этой системы состоит в том, что она расщепляется на множество независимых конечных подсистем линейных алгебраических уравнений относительно значений одной из компонент множества

$$
X_{k}=\left\{x_{j}=\psi_{k-j m, 1+j n}, k-j m \geqslant 1, j n \geqslant 1, j=0,1, \ldots,\left[\frac{k}{m}\right]-1\right\},
$$

где $x_{j}=0$ для всех $j<0$. Для фиксированных $k, m, n$ система линейных алгебраических уравнений, соответствующая (4.2), имеет трехдиагональньй вид

$$
\pm i x_{j}=\lambda A_{j} x_{j+1}+\bar{\lambda} B_{j} x_{j-1}
$$

с некоторыми положительными $A_{j}$ и $B_{j}$. Но хорошо известно [16], что

$$
D_{N}=\operatorname{det}\left(\begin{array}{cccccc} 
\pm i I & \lambda A_{1} & 0 & \ldots & 0 & 0 \\
\lambda^{*} B_{1} & \pm i I & \lambda A_{2} & \ldots & 0 & 0 \\
\ldots \ldots & \ldots & \ldots & \ldots & \ldots & \ldots \\
0 & 0 & 0 & \ldots & \lambda^{*} B_{N-1} & \pm i I
\end{array}\right) \neq 0
$$

где матричные элементы суть $(k \times k)$-блоки, $\lambda$ и $\lambda^{*}$ являются эрмитово сопряженными $(k \times k)$-матрищами, а $I$ является единичной матрицей в $\mathbb{C}^{M}$.

Согласно теореме Гершгорина [16] гамильтонианы (4.1) относительно ограничены оператором $\Lambda=c_{\Lambda}\left\{\left(a_{1}^{\dagger} a_{1}\right)^{M}\left(a_{2}^{\dagger} a_{2}\right)^{M}+1\right\}, M \geqslant m+n$, и относительная верхняя гранища гамильтониана $H$ убьвает при $c_{D} \rightarrow \infty$. Следовательно, все формальные генераторы с вполне положительными частями (3.1) и гамильтоновой частью (4.1) регулярны.

2. Такое же утверждение верно для гамильтонианов $H=H_{\text {s.a. }}+H_{s}$,

$$
H_{\text {s.a. }}=\lambda\left(a_{1}^{\dagger}\right)^{m_{1}} a_{1}^{n_{1}} \cdots a_{N}^{m_{N}}\left(a_{N}^{\dagger}\right)^{n_{N}}+\lambda^{*} a_{1}^{m_{1}}\left(a_{1}^{\dagger}\right)^{n_{1}} \cdots\left(a_{N}^{\dagger}\right)^{m_{N}} a_{N}^{n_{N}},
$$

действуюших в тензорном произведении $\mathscr{C}\left(\ell_{2}^{\otimes N} \otimes \mathbb{C}^{M}\right)$, где $\lambda$ и $\lambda^{*}$ являются эрмитово сопряженными $(M \times M)$-матрищами, $\sum m_{k}+n_{k}=K$, и $H_{s}$ - любой симметричный оператор, мажорируемый $\Lambda$, такой, что $\exists c \in \mathbb{R}: i\left[H_{s}, \Lambda\right] \leqslant c \Lambda$. Доказательство самосопряженности $H_{\text {s.a. }}$ основано на аналогичной факторизации блочных матрищ $\left\{\psi_{k_{1}, \ldots, k_{N}}\right\} \in \ell_{2}^{\otimes N} \otimes \mathbb{C}^{M}$ и на редукции однородной системы линейных алгебраических уравнений к множеству конечномерных линейных уравнений с невырожденными трехдиагональными $(M \times M)$-блочньми матрицами (4.3). Как и в предыдущем случае, представление взаимодействия порождается самосопряженным оператором $H_{\text {s.a. }}$, мажорируемым диагональньм оператором $\Lambda$ при $M \geqslant \sum\left(m_{k}+n_{k}\right)$, а гамильтониан $H_{s}$ входит в $\mathscr{L}_{0}(\cdot)$.

3. Рассмотрим физический пример [11] генератора $\mathscr{L}(\cdot)$, действующего в $\mathscr{B}\left(\ell_{2} \otimes\right.$ $\left.\ell_{2} \otimes \mathbb{C}^{2}\right)$ с вполне положительной частью (3.1) и гамильтонианом

$$
\widehat{H}=E i\left(a_{1}^{\dagger}-a_{1}\right)+\frac{\chi}{2} i\left(a_{1}^{\dagger 2} a_{2}-a_{1}^{2} a_{2}^{\dagger}\right)+\omega \sigma_{+} \sigma_{-}+\eta i\left(a_{2} \sigma_{+}-a_{2}^{\dagger} \sigma_{-}\right),
$$

где $E, \chi, \eta, \omega$ и $a_{2}-$ физические постоянные, $\sigma_{ \pm}-$эрмитово сопряженные $(2 \times 2)$-матрицы, а вполне положительная часть генератора имеет вид

$$
\Phi(B)=2 \gamma_{1} a_{1}^{\dagger} B a_{1}+2 \gamma_{2} a_{2}^{\dagger} B a_{2}+2 \kappa \sigma_{+} B \sigma_{-} .
$$


Коэффициенты $\gamma_{1}, \gamma_{2}$ и $\kappa$ - положительные константы [11]. Гамильтониан (4.5) может быть представлен в виде $(4.3)$ с $k=2, K=3, \lambda=I, H_{\text {s.a. }}=\frac{\chi}{2} i\left(a_{1}^{\dagger 2} a_{2}-a_{1}^{2} a_{2}^{\dagger}\right)$ и $H_{s}=H-H_{\text {s.a. }}$ Вполне положительная часть имеет вид (3.1). Следовательно, генератор (4.5), (4.5') регулярен. Этот пример являлся модельным при разработке изложенной вьше теории.

4. Кинетическая стадия эволюции некоторой квантовой системы, взаимодействующей с окружением, описывается в [17] с помощью следующего уравнения марковской эволюции в шрёдингеровском представлении:

$$
\begin{aligned}
\frac{\partial \sigma}{\partial t}= & -i \omega\left[H\left(a^{\dagger}, a\right), \sigma\right]+\Gamma_{2}\left(n_{2}+1\right)\left\{\left[a^{2} \sigma,\left(a^{\dagger}\right)^{2}\right]+\left[\left(a^{\dagger}\right)^{2}, \sigma a^{2}\right]\right\} \\
& +\Gamma_{2} n_{2}\left\{\left[\left(a^{\dagger}\right)^{2} \sigma, a^{2}\right]+\left[a^{2}, \sigma\left(a^{\dagger}\right)^{2}\right]\right\}
\end{aligned}
$$

где самосопряженный оператор $H=H\left(a^{\dagger}, a\right)$ является конечньм симметричньм полиномом от операторов $a^{\dagger}$ и $a$ не вьше четвертого порядка. Величины $\Gamma_{2}=\pi K^{2} g_{2}$, $n_{2}=n(2 \omega), K=K(2 \omega), g_{2}=g(2 \omega)$ являются скалярными функциями.

Соответствующее двойственное вполне положительное отображение $\Phi(\cdot)$ для генератора КДП в гейзенберговском представлении действует следующим образом:

$$
\Phi(B)=2 \Gamma_{2}\left(\left(n_{2}+2\right)\left(a^{\dagger}\right)^{2} B a^{2}+n_{2} a^{2} B\left(a^{\dagger}\right)^{2}\right) .
$$

Этот случай весьма прост: $H_{\text {int }}=H+\Lambda$ и $\Lambda=c\left(a^{\dagger}\right)^{2} a^{2}$, где значение $c$ достаточно велико: $\Lambda \geqslant \Phi(I)$ и $\|\Lambda h\| \geqslant 2\|H h\|$, так что $H_{\text {int }}$ является самосопряженным оператором, если $H$ является самосопряженным. Следовательно, генератор приведенного вьше уравнения марковской эволюции регулярен.

5. Предыдущий пример может быть обобщен следующим образом. Положим $\Phi_{m}^{+}(B)$ $=\left(a^{\dagger}\right)^{m} B a^{m}, \Phi_{m}^{-}(B)=a^{m} B\left(a^{\dagger}\right)^{m}, \Lambda_{n}=\left(\left(a^{\dagger} a\right)^{n}+I\right) \lambda$. Тогда $\Lambda_{n} \psi_{N}=\lambda\left(N^{n}+1\right) \psi_{N}$,

$$
\begin{gathered}
\Phi_{m}^{ \pm}(I) \psi_{N}=\left(\frac{(N \mp m) !}{N !}\right)^{\mp 1} \psi_{N}, \quad \Phi_{m}^{ \pm}(\Lambda) \psi_{N}=(N \mp m)^{n}\left(\frac{(N \mp m) !}{N !}\right)^{\mp 1} \psi_{N} \\
\left(\Phi_{m}^{ \pm}\left(\Lambda_{n}\right)-\Lambda_{n} \circ \Phi_{m}^{ \pm}(I)\right) \psi_{N}=\lambda\left(\frac{(N \mp m) !}{N !}\right)^{\mp 1}\left((N \mp m)^{n}-N^{n}\right) \psi_{N}
\end{gathered}
$$

для $N$-частичной компоненты $\psi_{N}$ вектора $\psi=\left\{\psi_{0}, \psi_{1}, \ldots\right\} \in \ell_{2}$. Поэтому для любого генератора с вполне положительной частью

$$
\Phi(B)=\sum_{k}\left\{c_{k}^{+} \Phi_{m_{k}}^{+}(B)+c_{k}^{-} \Phi_{n_{k}}^{-}(B)\right\}
$$

с положительными матричнозначными коэффициентами $c_{k}^{ \pm} \in \mathbb{C}^{M} \otimes \mathbb{C}^{M}$ условие $\mathscr{L}_{0}(\Lambda)$ $\leqslant c \Lambda$ вьполнено, если вьполнено условие баланса:

$$
\sup _{N \geqslant 1} \sum_{k}\left\{c_{k}^{+} \frac{N !}{\left(N-m_{k}\right) !}\left[\left(1-\frac{m_{k}}{N}\right)^{n}-1\right]+c_{k}^{-} \frac{\left(N+n_{k}\right) !}{N !}\left[\left(1+\frac{n_{k}}{N}\right)^{n}-1\right]\right\}<c I .
$$

6. В статье [18] рассматриваются примеры генераторов в $\mathscr{H}=\ell_{2} \otimes \ell_{2}$ с вполне положительной компонентой $\Phi(B)=a_{1} a_{2}^{\dagger} B a_{2} a_{1}^{\dagger}$. Докажем, что для генераторов с коэффициентами

$$
\Phi(B)=a_{1}^{L}\left(a_{2}^{\dagger}\right)^{M} B a_{2}^{M}\left(a_{1}^{\dagger}\right)^{L}, \quad H=H_{2}+H_{\text {s.a. }}\left(a^{\dagger}, a\right)
$$

при $L=M=1$ существует $\Lambda$-пара с любым самосопряженным полиномом $H_{\text {s.a. }}\left(a^{\dagger}, a\right)$ конечного порядка. Рассмотрим генератор

$$
\mathscr{L}_{0}(B)=a_{1}^{L}\left(a_{2}^{\dagger}\right)^{M} B a_{2}^{M}\left(a_{1}^{\dagger}\right)^{L}-B \circ a_{1}^{L}\left(a_{1}^{\dagger}\right)^{L}\left(a_{2}^{\dagger}\right)^{M} a_{2}^{M} .
$$


Лемма 4.1. При $L=M=1$ для любого $N \geqslant 2$ существуют $\lambda, \lambda_{k} \geqslant 0$ такие, чmo

$$
\Lambda_{N}=\lambda I+\sum_{k=0}^{N} \lambda_{k}\left(a_{1}^{\dagger}\right)^{N-k} a_{1}^{N-k}\left(a_{2}^{\dagger}\right)^{k} a_{2}^{k} \geqslant \Phi(I), \quad \mathscr{L}_{0}\left(\Lambda_{N}\right) \leqslant c_{N} \Lambda_{N}
$$

для некоторой постоянной $c_{N}$.

ДокАЗАТЕЛЬСтво. Заметим, что в общем случае

$$
\begin{aligned}
\mathscr{L}_{0}\left(\left(a_{1}^{\dagger}\right)^{m} a_{1}^{m}\left(a_{2}^{\dagger}\right)^{n} a_{2}^{n}\right)= & m L\left(a_{1}^{\dagger}\right)^{L+m-1} a_{1}^{L+m-1}\left(a_{2}^{\dagger}\right)^{n+M} a_{2}^{n+M} \\
& -n M\left(a_{1}^{\dagger}\right)^{L+m} a_{1}^{L+m}\left(a_{2}^{\dagger}\right)^{n+M-1} a_{2}^{n+M-1}+\text { (н.п.), }
\end{aligned}
$$

где (н.п.) - члены, имеющие на две единицы более низкий порядок. Следовательно,

$$
\begin{gathered}
\mathscr{L}_{0}\left(\Lambda_{N}\right)=-\sum_{k=0}^{N-1} \nu_{k} \cdot\left(a_{1}^{\dagger}\right)^{L+N-k-1} a_{1}^{L+N-k-1}\left(a_{2}^{\dagger}\right)^{k+M} a_{2}^{k+M}+(\text { н.п. }), \\
\nu_{k}=M(k+1) \lambda_{k+1}-L(N-k) \lambda_{k},
\end{gathered}
$$

где все старшие члены имеют порядок $2(L+M+N-1)$ и входят в сумму со знаком минус, если $\nu_{k}>0$, т.е. если

$$
\lambda_{k+1}>\frac{\lambda_{k}(N-k) L}{(k+1) M}
$$

Младшие члены имеют порядок $2(L+M+N-2)$ равный $2 N$, если $L=M=1$. Таким образом,

$$
\left.\mathscr{L}_{0}\left(\Lambda_{N}\right) \leqslant \text { (н.п. }\right)-\nu \sum_{k=0}^{N-1}\left(a_{1}^{\dagger}\right)^{L+N-k-1} a_{1}^{L+N-k-1}\left(a_{2}^{\dagger}\right)^{k+M} a_{2}^{k+M},
$$

где $\nu=\min _{k}\left\{\nu_{k}\right\}>0$. Очевидно, что оператор $\Lambda_{N}$ имеет порядок $2 N$ и

$$
\Lambda_{N} \geqslant \lambda I+\lambda_{0}\left(a_{1}^{\dagger}\right)^{N} a_{1}^{N}+\lambda_{N}\left(a_{2}^{\dagger}\right)^{N} a_{2}^{N} \geqslant \Phi(I)=a_{1} a_{1}^{\dagger} a_{2}^{\dagger} a_{2}
$$

если коэффициенты $\lambda, \lambda_{0}, \lambda_{N}$ выбраны достаточно большими. Следовательно, при $L=$ $M=1$ младшие члены можно мажорировать оператором $c_{N} \Lambda_{N}$ для всех достаточно больших $c_{N}$. Поэтому если выполнено (4.9), то существует положительная постоянная $c_{N}$ такая, что, с одной стороны, $\mathscr{L}_{0}\left(\Lambda_{N}\right) \leqslant c_{N} \Lambda_{N}$, a, с другой, в силу произвольности $N$ оператор $c_{N} \Lambda_{N}$ мажорирует с произвольно малой относительной верхней гранищей любой заданный полином конечного порядка $H_{\text {s.a. }}\left(a^{\dagger}, a\right)$. Поэтому $\left\{\Lambda_{N}, H_{\text {int }}=\right.$ $\left.\Lambda_{N}+H_{\text {s.a. }}\right\}$ является $\Lambda$-парой для формального генератора $\mathscr{L}(\cdot)$ с коэффициентами (4.7). Нам удалось доказать регулярность $\mathscr{L}(\cdot)$ в случае $L=M=1$ (см. [18]). Вопрос о конструкции $\Lambda$-пары в общем случае остается открытым. 
5. Достаточные условия нарушения консервативности. Условие, необходимое и достаточное для того, чтобы минимальное решение было унитальным, состоит в слабой сходимости к нулю монотонно убывающей последовательности ограниченных положительных операторов

$$
Q_{\varepsilon}^{n}(I) \rightarrow 0, \quad Q_{\varepsilon}(X) \stackrel{\text { def }}{=} \int_{0}^{\infty} d t e^{-\varepsilon t} W_{t}^{*} \Phi(X) W_{t}
$$

при некотором $\varepsilon>0$ (см. [3]). Следовательно, существование положительного оператора $X,\|X\| \leqslant I$, такого, что $Q_{\varepsilon}(X) \geqslant X$ для некоторого $\varepsilon>0$, является достаточным для нарушения унитальности, поскольку в этом случае $Q_{\varepsilon}^{n}(I)$ не сходится к нулю5:

$$
I \geqslant Q_{\varepsilon}(I) \geqslant Q_{\varepsilon}^{n}(I) \geqslant Q_{\varepsilon}^{n}(X) \geqslant Q_{\varepsilon}(X) \geqslant X \neq 0
$$

В [4], [19] доказано, что для вполне положительного отображения

$$
\widetilde{Q}_{\varepsilon}(X)=Q_{\varepsilon}(I)^{-1} Q_{\varepsilon}(X) Q_{\varepsilon}(I)^{-1}
$$

и положительного самосопряженного оператора $S=\left(Q_{\varepsilon}(I)^{-1}-I\right)^{-1}$ вьполнено неравенство

$$
Q_{\varepsilon}^{n+1}(I) \geqslant\left(\widetilde{Q}_{\varepsilon}(I)^{-1}+\widetilde{Q}_{\varepsilon}\left(S^{-1}\right)^{-1}+\cdots+\widetilde{Q}_{\varepsilon}^{n}\left(S^{-1}\right)^{-1}\right)^{-1} .
$$

Поэтому расходимость ряда $\widetilde{Q}_{\varepsilon}(I)^{-1}+\widetilde{Q}_{\varepsilon}\left(S^{-1}\right)^{-1}+\cdots+\widetilde{Q}_{\varepsilon}^{n}\left(S^{-1}\right)^{-1}+\cdots$ на каком-либо плотном подмножестве в $\mathscr{H}$ является необходимым условием унитальности минимальной КДП. Это условие, однако, не дает простого ответа на вопрос, какой оператор можно использовать в качестве оператора $X$ в условии $(5.2)$, поэтому здесь мы рассмотрим более простой критерий, охватьвающий случаи нарушения консервативности за счет нарушения самосопряженности гамильтоновой части генератора.

Заметим, что эрмитова структура полинома (3.2) не гарантирует его самосопряженность. Например, симметричньй гамильтониан третьего порядка

$$
H=\frac{i}{2 \sqrt{2}}\left\{\left(1+\frac{1}{2}\left(a+a^{\dagger}\right)^{2}\right)\left(a-a^{\dagger}\right)+\left(a-a^{\dagger}\right)\left(1+\frac{1}{2}\left(a+a^{\dagger}\right)^{2}\right)\right\}
$$

не является самосопряженным оператором в $\ell_{2}$, поскольку он унитарно эквивалентен симметричному оператору $\widehat{H}=i\left(\left(1+x^{2}\right) \partial_{x}+x\right)$ в $\mathscr{L}_{2}(\mathbb{R})$, имеющему нормированньй собственный вектор $\varphi_{\mu}$ такой, что

$$
\widehat{H} \varphi_{\mu}=-i \mu \varphi_{\mu}, \quad \varphi_{\mu}(x)=\frac{\varphi_{0}}{\sqrt{1+x^{2}}} e^{-\mu \operatorname{arctg}(x)} \in \mathscr{L}_{2}(\mathbb{R}), \quad \mu>0, \quad \varphi_{0}=\sqrt{\frac{\operatorname{sh} \pi \mu}{\mu}} .
$$

\footnotetext{
${ }^{5}$ Например, если $\Phi(B)=a^{2} B\left(a^{\dagger}\right)^{2}$ и $H=0$, то $\Phi(I)=\left(a^{\dagger} a\right)^{2}+4 a^{\dagger} a+4 I=p\left(a^{\dagger} a\right)$ и $Q_{\varepsilon}(I)=\left(I+\varepsilon p\left(a^{\dagger} a\right)^{-1}\right)^{-1}=q_{\varepsilon}\left(a^{\dagger} a\right)$. Учитывая, что $\Phi\left(q_{\varepsilon}\left(a^{\dagger} a\right)\right)=p\left(a^{\dagger} a\right) q_{\varepsilon}\left(a^{\dagger} a+2\right)$, получаем $Q_{\varepsilon}^{n}(I)=\prod_{k=0}^{n} q_{\varepsilon}\left(a^{\dagger} a+2 k\right)$. Эта последовательность операторов, очевидно, не сходится к нулю: из неравенства $(1+x)^{-1} \geqslant e^{-x}$ при $x>0$ имеем равномерную по $n$ оценку $Q_{\varepsilon}^{n}(I) \geqslant \exp \left\{-\varepsilon \sum_{k=1}^{\infty} p\left(a^{\dagger} a+2 k\right)^{-1}\right\} \geqslant \exp \left\{-\varepsilon \sum_{k=1}^{\infty}(2 k)^{-2}\right\}>0$.
} 
Следовательно, симметричные операторы $H$ и $\widehat{H}$ имеют нетривиальньй индекс дефекта, а оператор $\pi_{\mu}=\left|\varphi_{\mu}\right\rangle\left\langle\varphi_{\mu}\right|$ является проектором на дефектное подпространство оператора $H$. Этот оператор и является кандидатом для подстановки в (5.2). Отметим также, что показатель экспоненты равен времени ухода оператора координаты на бесконечность из точки $x$ для динамической системы с гамильтонианом $H(x, p)=\left(1+x^{2}\right) p+x$. Ниже мы рассмотрим условия, которые обеспечивают нарушение свойства унитальности.

В этом пункте мы предполагаем, что $-G$ - генератор сильно непрерывной сжимающей полугрупшы $W_{t}=e^{-G t}$ и либо $\operatorname{dom} G^{N} \subseteq \operatorname{dom} \Phi(I)^{1 / 2}$ для некоторого $N \geqslant 1$, либо существует $\Lambda \geqslant \Phi(I)$ и для $G$ вьполнено условие (2.2). Положим $\mathscr{D}=\operatorname{dom} G^{N}$ в первом случае и $\mathscr{D}=\operatorname{dom} \Lambda^{1 / 2}$ во втором; оператор $H$ симметричный на $\mathscr{D}$. Очевидно, что $\pi \mathscr{D}=\varnothing$.

ТЕОРема 5.1. Если коэффициенты генератора $\mathscr{L}(\cdot)$ удовлетворяют условиям на область определения $\mathscr{D}$ и существуют положительный оператор $X,\|X\| \leqslant 1$, $u \varepsilon>0$ такие, что

$$
\mathscr{L}_{*}(X)[\psi] \geqslant \varepsilon X_{*}[\psi] \quad \forall \psi \in \mathscr{D},
$$

то минимальная КДП с формальным генератором $\mathscr{L}(\cdot)$ не консервативна.

ДокаЗАТЕЛЬСтво. Покажем, что из (5.5) следует неравенство $Q_{\varepsilon}(X) \geqslant X$. Заметим, что из $(5.5)$ следует $\Phi(X)_{*} \geqslant\left(\varepsilon X+G^{*} X+X G\right)_{*}$ на $\mathscr{D}$. Поэтому для любого $\psi \in \mathscr{D}$ имеем

$$
e^{-\varepsilon t} \Phi_{*}(X)\left[\psi_{t}\right] \geqslant e^{-\varepsilon t}\left(\varepsilon\left(\psi_{t}, X \psi_{t}\right)+\left(G \psi_{t}, X \psi_{t}\right)+\left(X \psi_{t}, G \psi_{t}\right)\right)=-\frac{d}{d t}\left(e^{-\varepsilon t}\left\|X \psi_{t}\right\|^{2}\right),
$$

где $\psi_{t}=W_{t} \psi \in \mathscr{D}$. Интегрируя это неравенство, получим

$$
\int_{0}^{t} e^{-\varepsilon \tau} \Phi(X)_{*}\left[\psi_{\tau}\right] d \tau \geqslant(\psi, X \psi)-e^{-\varepsilon t}\left(\psi_{t}, X \psi_{t}\right) .
$$

Предельньй переходпри $t \rightarrow \infty$ в (5.6) обеспечивает сходимость правой части к $(\psi, X \psi)$, а левая часть сходится к $\left(\psi, Q_{\varepsilon}(X) \psi\right)$ для любого $\psi \in \mathscr{D}$ по определению $Q_{\varepsilon}(\cdot)$. Поскольку множество $\mathscr{D}$ плотно в $\mathscr{H}$ и отображение $Q_{\varepsilon}(\cdot)$ является ограниченным, это неравенство эквивалентно условию (5.2), что противоречит необходимому условию унитальности.

Естественным кандидатом на роль оператора $X$ в условии (5.5) является проектор на дефектное подпространство оператора $H$, если такое подпространство существует.

ТЕОРема 5.2 (см. [20]). Пусть Н является плотно определенным симметричныц оператором. Предположим, что он имеет нетривиальное дефектное подпространство $\mathscr{H}_{d}=\left\{\psi: H^{*} \psi_{\mu}=-i \mu \psi_{\mu}\right\}$ и $\pi_{\mu}$ является проектором на $\mathscr{H}_{d}$. Если, кроме того, существует постоянная $\varepsilon>0$ такая, что

$$
\Phi\left(\pi_{\mu}\right)_{*}[\psi]-\operatorname{Re}\left(\Phi(I) \psi, \pi_{\mu} \psi\right) \geqslant-(2-\varepsilon)\left\|\pi_{\mu} \psi\right\|^{2} \quad \forall \psi \in \mathscr{D},
$$

то (5.5) выполнено, а необходимое условие унитальности (5.1) нарушено. 
ДокАЗАТЕЛЬСтво. Докажем, что из (5.7) следует (5.6) в следующей форме:

$$
\mathscr{L}\left(\pi_{\mu}\right)_{*}[\psi] \geqslant \varepsilon\left\|\pi_{\mu} \psi\right\|^{2} \quad \forall \psi \in \mathscr{D} .
$$

Поскольку оператор $H$ симметричен на $\mathscr{D}$ и $H^{*} \pi_{\mu}=-i \mu \pi_{\mu}$, то из (5.7) очевидно следует, что

$$
\begin{aligned}
\mathscr{L}\left(\pi_{\mu}\right)_{*}[\psi] & =\Phi\left(\pi_{\mu}\right)_{*}[\psi]-\operatorname{Re}\left(\Phi(I) \psi, \pi_{\mu} \psi\right)+i\left(\left(H \psi, \pi_{\mu} \psi\right)-\left(\pi_{\mu} \psi, H \psi\right)\right) \\
& =\Phi\left(\pi_{\mu}\right)_{*}[\psi]-\operatorname{Re}\left(\Phi(I) \psi, \pi_{\mu} \psi\right)+2 \mu\left\|\pi_{\mu} \psi\right\|^{2} \geqslant \varepsilon\left\|\pi_{\mu} \psi\right\|^{2},
\end{aligned}
$$

и поэтому выполнено неравенство (5.6).

В качестве примера рассмотрим КДП с гамильтонианом (5.3) и вполне положительной частью $\Phi(B)=\frac{\lambda}{2}\left(a^{\dagger}-a\right) B\left(a-a^{\dagger}\right)$. В этом случае левая часть условия $(5.7)$ имеет вид

$$
\begin{aligned}
\Delta & =\Phi\left(\pi_{\mu}\right)_{*}[\psi]-\operatorname{Re}\left(\Phi(I) \psi, \pi_{\mu} \psi\right) \\
& =\frac{\lambda}{2}\left\{\left|\left(\left(a^{\dagger}-a\right) \psi_{\mu}, \psi\right)\right|^{2}-\operatorname{Re}\left\{\left(\psi,\left(a^{\dagger}-a\right)^{2} \psi_{\mu}\right) \cdot\left(\psi_{\mu}, \psi\right)\right\}\right\} \\
& \geqslant \frac{\lambda}{2}\left\{\left|\left(\left(a^{\dagger}-a\right) \psi_{\mu}, \psi\right)\right|^{2}-\left|\left(\psi,\left(a^{\dagger}-a\right)^{2} \psi_{\mu}\right)\right| \cdot\left|\left(\psi_{\mu}, \psi\right)\right|\right\},
\end{aligned}
$$

или в унитарно эквивалентном $\mathscr{L}_{2}$-представлении

$$
\begin{gathered}
\Delta \geqslant \lambda\left\{\left|\left(\psi_{\mu}, K \psi\right)\right|^{2}-\left|\left(\psi_{\mu}, M \psi\right)\right| \cdot\left|\left(\psi_{\mu}, \psi\right)\right|\right\}>-\mu_{*} \lambda\left|\left(\psi_{\mu}, \psi\right)\right|^{2}=-\lambda \mu\left\|\pi_{\mu} \psi\right\|^{2}, \\
K_{m} u(x)=\frac{x+\mu}{x^{2}+1}, \quad M_{m} u(x)=\frac{2 x^{2}+4 \mu x-1}{\left(x^{2}+1\right)^{2}},
\end{gathered}
$$

поскольку при $\mu \rightarrow \infty \mu_{*} / \mu=\max _{x}\left|M \mu_{*}(x)\right| / \mu \rightarrow 1$. Таким образом, согласно (5.7) условие консервативности нарушено при всех $\lambda \in(0,2]$. Классическое необходимое и достаточное условие Феллера для взрьва одномерной диффузии со скоростью сноса $1+x^{2}[21]$ не накладьвает никаких ограничений на величину коэффициента диффузии. Поэтому следует ожидать, что существуют более сильные достаточные условия взрыва для КДП, допускающие $\lambda \in(2, \infty)$.

6. Заключение. Заканчивая статью, отметим две проблемы, решение которых представляет большой теоретический интерес.

Как мы видели в п. 5, генератор марковской эволюции может оказаться нерегулярным, но это не исключает существование минимальной КДП. В работе [22] все унитальные расширения минимальной КДП были параметризованы с помощью расширения ее минимальной резольвенты, однако связь этих расширений с условиями физического или вероятностного характера пока остается невыясненной.

В классической теории случайных процессов инфинитезимальный оператор для марковской полугруппы и краевые условия для стохастических процессов вытекают из формулы Дьнкина [23], [24], в которой центральную роль играет понятие момента первого достижения. В квантовой теории решения квантовых стохастических дифференциальных уравнений также позволяют выразить КДП через математические ожидания, и хотя существует немало математических работ, посвященных операторньм обобщениям конструкции момента первого достижения, попытки перенести формулу Дьнкина для квантовых стохастических процессов, по-видимому, не предпринимались. 


\section{СПИСОК ЦИТИРОВАННОЙ ЛИТЕРАТУРЫ}

[1] Гарсиа Х. С. О структуре конуса нормальных неограниченных вполне положительных отображений // Матем. заметки. 1999. Т. 65. № 2. С. 194-205.

[2] Davies E. B. Quantum dynamical semigroups and neutron diffusion equation // Rep. Math. Phys. 1979. V. 11. № 2. P. 169-188.

[3] Chebotarev A. M. Necessary and sufficient conditions for conservativity of a dynamical semigroup // J. Soviet Math. 1991. V. 56. № 5. P. 2697-2719.

[4] Чеботарев А. М. О достаточных условиях консервативности минимальной динамической полугруппы // Матем. заметки. 1992. Т. 52. №4. С. 112-127.

[5] Holevo A. S. Stochastic differential equations in Hilbert space and quantum Markovian evolutions // Probability Theory and Mathematical Statistics. Proc. of the 7th Japan-Russian Symposium. Singapore: World Sci., 1996. P. 122-131.

[6] Chebotarev A. M., Garcia J. C., Quezada R. B. A priori estimates and existence theorems for the Lindblad equation with unbounded time-dependent coefficients // Recent Trends in Infinite Dimensional Non-Commutative Analysis. V. 1035. Kokyuroku: Publ. Res. Inst. Math. Sci., 1998. P. 44-65.

[7] Chebotarev A. M., Fagnola F. Sufficient conditions for conservativity of minimal quantum dynamical semigroups // J. Funct. Anal. 1998. V. 153. № 2. P. 382-404.

[8] Bratteli O., Robinson D. W. Operator Algebras and Quantum Statistical Mechanics. V. I. Berlin: Springer-Verlag, 1981.

[9] Kraus K. General state changes in quantum theory // Ann. Phys. 1971. V. 64. P. 311-335.

[10] Kato T. Perturbation Theory for Linear Operators. Berlin: Springer-Verlag, 1976.

[11] Schack R., Brun T.A., Percival I. C. Quantum state diffusion with a moving basis: computing quantum optical spectra // Phys. Rev. A. 1996. V. 53. P. 2694.

[12] Zoller P., Gardiner C. W. Quantum noise in quantum optics: The stochastic Schödinger equation // Lecture Notes for the Les Houches Summer School LXIII on Quantum Fluctuations in July 1995 / ed. E. Giacobino, S. Reynaud: Elsevier Sci. Publ. (B.V.), 1997.

[13] Wiseman H. M., Vaccaro J. A. Maximally robust unravelings of quantum master equations // Phys. Lett. A. 1998. V. 250. P. 241-248.

[14] Kist T. B. L., Orszag M., Brun T. A., Davidovich L. Physical interpretation of stochastic Schrödinger equations in cavity QED // LANL E-print quant-ph/9805027.

[15] Reed M., Simon B. Methods of Modern Mathematical Physics. V. 1. Functional Analysis. New York: Acad. Press, 1981.

[16] Bellman R. Introduction to Matrix Analysis. New York: McGraw-Hill, 1978.

[17] Kilin D., Schreiber M. Influence of phase-sensitive interaction on the decoherence process in molecular systems // LANL E-print quant-ph/9707054.

[18] Lanz L., Melsheimer O., Vacchini B. Subdynamics through Time Scales and Scattering Maps in Quantum Field Theory // Proceedings of the Third International Conference on Quantum Communication and Measurement 1996 (Hakone, Japan), 1997.

[19] Chebotarev A. M. Lectures on Quantum Probability. Mexico: SMM, 2000.

[20] Chebotarev A. M., Shustikov S. Yu. On a condition sufficient for violation of unitality // VINITI 09.06.2000 No 1645-B00.

[21] McKean H. P. Stochastic Integrals. New York: Acad. Press, 1969.

[22] Чеботарев А. М. О максимальной $C$ *-алгебренулей вполне положительного отображения и границе динамической полугруппы // Матем. заметки. 1994. Т. 56. №6. С. 88-105.

[23] Dynkin E. B. Markov Processes. Berlin: Springer-Verlag, 1965.

[24] Grigor'yan A. Analytic and geometric background of recurrence and non-explosion of the Brownian motion on Riemannian manifolds // Bull. Amer. Math. Soc. 1999. V. 36. № 2. P. 135-249.

Московский государственный университет им. М. В. Ломоносова 